\title{
两项时间混合分数阶扩散波动方程的有限元高精度 分析
}

\author{
魏亚冰 ${ }^{1,2}$ ，赵艳敏 ${ }^{1}$ ，唐贻发 ${ }^{3,4^{*}}$ ，王芬玲 ${ }^{1}$ ，史争光 ${ }^{5}$ ，李匡郢 ${ }^{6}$ \\ 1. 许昌学院数学与统计学院, 许昌 461000 , 中国 \\ 2. 郑州大学数学与统计学院, 郑州 450001, 中国 \\ 3. 中国科学院数学与系统科学研究院, 北京 100190 , 中国 \\ 4. 中国科学院大学数学科学学院, 北京 100049 , 中国 \\ 5. 西南财经大学经济数学学院, 成都 611130 , 中国 \\ 6. McDougall School of Petroleum Engineering, The University of Tulsa, Tulsa, OK 74104, USA \\ * 通信作者. E-mail: tyf@lsec.cc.ac.cn
}

收稿日期: 2018-03-27；接受日期: 2018-04-08; 网络出版日期: 2018-07-12

国家自然科学基金 (批准号: 11771438, 11101381, 11471296) 资助项目

摘要 基于空间方向的有限元方法和时间方向的 L1-CN 格式, 本文针对二维两项时间混合分数阶 扩散波动方程进行数值分析. 首先, 给出该方程的全离散逼近格式, 并证明其无条件稳定性. 然后, 严格证明 $L^{2}$ 模意义下的收敛结果和 $H^{1}$ 模意义下的超逼近结果 $O\left(h^{2}+\tau^{\min \left\{2-\alpha_{1}, 3-\alpha\right\}}\right)\left(0<\alpha_{1}<\right.$ $1,1<\alpha<2)$, 这里 $h$ 和 $\tau$ 分别表示空间和时间步长. 进一步地, 利用插值后处理技术导出 $H^{1}$ 模意 义下的整体超收玫结果。最后, 借助于数值算例进一步展示理论分析的正确性和高效性.

关键词分数阶扩散波动方程, 有限元方法, L1-CN 格式, 稳定性, 超逼近, 收玫和超收玫

\section{1 引言}

近年来, 分数阶偏微分方程模型已经渗透到许多不同的科学领域. 比如, 在生物学、物理学、医学、

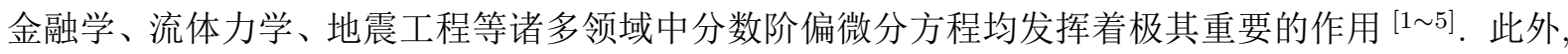
还有大量研究表明, 分数阶偏微分方程在描述物质的记忆和遗传等性质方面有着独特的优势. 基于分 数阶偏微分方程在这些领域中的广泛应用, 目前已有大量国内外研究者关注分数阶偏微分方程.

分数阶偏微分方程大致可分为 3 类: 时间分数阶偏微分方程、空间分数阶偏微分方程和时间 - 空 间分数阶偏微分方程, 本文重点关注时间分数阶偏微分方程. 对大多数时间分数阶偏微分方程来说, 其解析解不易求得. 即便可得到解析解, 其表达式中大多含有特殊函数, 并且这些特殊函数的计算相

\footnotetext{
引用格式: 魏亚冰, 赵艳敏, 唐贻发, 等. 两项时间混合分数阶扩散波动方程的有限元高精度分析. 中国科学: 信息科学, 2018, 48: 871-887, doi: 10.1360/N112017-00295

Wei Y B, Zhao Y M, Tang Y F, et al. High-accuracy analysis of finite-element method for two-term mixed timefractional diffusion-wave equations (in Chinese). Sci Sin Inform, 2018, 48: 871-887, doi: 10.1360/N112017-00295
} 
当困难, 因此求解时间分数阶偏微分方程的数值解和数值方法日益受到关注. 常用的求解时间分数阶

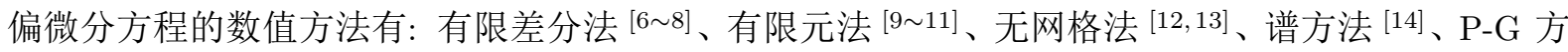
法 [15] 等.

目前, 对时间分数阶偏微分方程的数值研究主要集中在时间分数阶扩散方程和时间分数阶扩散波 动方程这两类方程. 例如, 对于单项时间分数阶扩散方程, 文献 [16] 分别利用空间方向的协调和非协 调混合有限元方法结合时间方向的经典 L1 格式, 给出单项时间分数阶扩散方程的无条件稳定的全离 散逼近格式, 并证明了原始变量 $u$ 在 $H^{1}$ 模意义下以及流量 $\boldsymbol{p}=\nabla u$ 在 $L^{2}$ 模意义下的空间方向整体 超收玫以及时间方向最优收玫阶为 $O\left(h^{2}+\tau^{2-\beta}\right), 0<\beta<1$. 文献 [17] 采用时空谱方法求解单项时间 分数阶扩散方程, 并得到了零初始条件和齐次边界条件下空间和时间方向的谱精度. 文献 [18] 提出了 一种隐式无网格方法, 用于求解单项时间分数阶扩散方程. 文献 [19] 基于 Jacobi 多项式, 利用空间方 向 Legendre 谱方法和时间方向有限差分方法, 针对单项时间分数阶扩散方程做了稳定性和收玫性分 析. 文献 [20] 利用双线性元、Raviart-Thomas 元和 L1 时间步长方法, 建立了二维单项时间分数阶扩 散方程的 $H^{1}$-Galerkin 混合有限元近似格式, 然后证明其无条件稳定性, 并且给出了超逼近和超收敛 的结果. 以上这些均是关于单项时间分数阶扩散方程的数值研究, 同时, 还有一些是关于多项时间分数 阶扩散方程的数值分析. 比如, 文献 [21] 基于分段线性函数, 用标准 Galerkin 有限元方法给出多项时 间分数阶扩散方程的空间半离散格式, 并讨论了其稳定性和误差估计. 文献 [22] 利用谱方法对多项时 间分数阶扩散方程做了数值近似. 文献 [23] 基于空间方向上的有限元方法和时间方向上的经典 L1 逼 近方法, 建立二维多项时间分数阶扩散方程的全离散格式, 并利用双线性单元的性质和插值后处理技 术, 分析了空间方向的整体超收玫性和时间方向收玫性. 文献 [24] 采用空间方向非协调有限元方法和 时间方向改进的 L1 格式, 对多项时间分数阶扩散方程建立无条件稳定的全离散逼近格式, 并给出时 间最优误差估计和空间最优收敛速率.

此外, 关于时间分数阶扩散波动方程, 有以下一些研究. 文献 [25 33] 是针对单项时间分数阶扩 散波动方程做的一些数值分析. 例如, 文献 [25] 借助于空间二维单项时间分数阶扩散波动方程来模拟 常量 $\mathrm{Q}$ 波传播, 并利用 Grünwald-Letnikov 差分和中心差分近似计算分数阶导数, 获得了对应于每个 Fourier 分量的相位速度. 文献 [26] 构造单项时间分数阶扩散波动方程的全离散格式, 然后证明其解的 存在唯一性及无条件稳定性, 并给出了在 $L_{\infty}$ 模意义下的收玫性. 文献 [27] 利用交替方向隐式 (ADI) 正交样条配置方法来逼近二维单项时间分数阶扩散波动方程, 并给出了不同模意义下的最优精度. 文 献 [28] 利用微分方程与积分方程的等价性构造单项时间分数阶扩散波动方程的两个有限差分格式, 同 时还证明了它们在时间方向上具有一阶精度, 在空间方向上具有二阶精度. 文献 [29] 致力于研究单项 时间分数阶扩散波动方程的高阶差分方法, 并给出了逼近格式的无条件稳定性和 $L_{\infty}$ 模意义下的收玫 性. 文献 [30] 基于空间方向的分段线性 Galerkin 有限元方法和时间方向的卷积积分, 对具有非光滑初 值的单项时间分数阶扩散波动方程提出了两种全离散逼近格式. 文献 [31] 利用空间方向的 Galerkin 有限元方法和时间方向的 ADI 格式, 提出二维单项时间分数阶扩散波动方程的一种数值逼近格式, 并 严格证明其无条件稳定性和 $L^{2}$ 模意义下的收玫性. 文献 [32] 基于 Jacobi tau 谱方法和分数阶积分的 Jacobi 运算矩阵, 提出了求解二阶和四阶单项时间分数阶扩散波动方程以及具有阻尼项的时间分数阶 扩散波动方程的高效谱方法. 文献 [33] 基于空间方向有限元方法和时间方向 Crank-Nicolson 近似, 建 立二维单项时间分数阶扩散波动方程的全离散格式, 并对该近似方法做无条件稳定性分析. 然后, 给出 了 $H^{1}$ 模下空间和时间的收玫结果. 以上均为致力于单项时间分数阶扩散波动方程的研究, 文献 [34] 将考虑多项时间分数阶扩散波动方程. 文献 [34] 采用有限差分法和分数阶预测校正法来模拟多项时 间分数阶扩散波动方程, 并可将这些方法和技术扩展到具有分数阶 Laplace 算子的其他多项分数阶偏 
微分方程模型.

以上这些研究均是关于单项及多项时间分数阶扩散方程或时间分数阶扩散波动方程的, 然而, 单 一地使用这两类方程不能灵活、准确地来描述一些实际过程. 倘若将这两类方程有机结合, 即两项时 间混合分数阶扩散波动方程, 则可更为精确地对这些过程进行模拟. 因此, 本文主要考虑如下二维两 项时间混合分数阶扩散波动方程:

$$
\begin{cases}D_{t}^{\alpha_{1}} u(\boldsymbol{x}, t)+D_{t}^{\alpha} u(\boldsymbol{x}, t)-\Delta u(\boldsymbol{x}, t)=f(\boldsymbol{x}, t), & (\boldsymbol{x}, t) \in \Omega \times(0, T], \\ u(\boldsymbol{x}, t)=0, & (\boldsymbol{x}, t) \in \partial \Omega \times(0, T], \\ u(\boldsymbol{x}, 0)=u_{0}(\boldsymbol{x}), \quad u_{t}(\boldsymbol{x}, 0)=\tilde{u}_{0}(\boldsymbol{x}), & \boldsymbol{x} \in \Omega,\end{cases}
$$

其中 $\Omega \subset \mathbb{R}^{2}$ 是一个有界的凸多边形区域, 边界为 $\partial \Omega$, 我们假设 $\boldsymbol{x}=(x, y), u_{0}(\boldsymbol{x})$ 和 $f(\boldsymbol{x}, t)$ 为已知的 足够光滑的函数, 而 $D_{t}^{\alpha}, D_{t}^{\alpha_{1}}$ 分别是 $\alpha$ 和 $\alpha_{1}$ 阶的关于 $t$ 的左侧 Caputo 分数阶导数, 如文献 [35] 中 所定义:

$$
\begin{aligned}
& D_{t}^{\alpha_{1}} u(\boldsymbol{x}, t)=\frac{1}{\Gamma\left(1-\alpha_{1}\right)} \int_{0}^{t} \frac{\partial u(\boldsymbol{x}, s)}{\partial s} \frac{\mathrm{d} s}{(t-s)^{\alpha_{1}}}, \quad 0<\alpha_{1}<1, \\
& D_{t}^{\alpha} u(\boldsymbol{x}, t)=\frac{1}{\Gamma(2-\alpha)} \int_{0}^{t} \frac{\partial u^{2}(\boldsymbol{x}, s)}{\partial s^{2}} \frac{\mathrm{d} s}{(t-s)^{\alpha-1}}, \quad 1<\alpha<2,
\end{aligned}
$$

其中 $\Gamma(\cdot)$ 表示 Gamma 函数.

到目前为止, 关于两项时间混合分数阶扩散波动方程的数值分析研究还十分有限, 相关的高精度 数值算法更是稀缺. 本文尝试借助于双线性有限元方法结合 L1-CN 格式来给出两项时间混合分数阶 扩散波动方程的数值分析. 众所周知, 有限元方法是求解偏微分方程的一种快速高效的数值方法, 而 超收玫技术是提高有限元数值近似精度的有效方法 [36 38]. 首先, 基于空间方向双线性有限元方法和 时间方向 L1-CN 格式, 本文对两项时间混合分数阶扩散波动方程建立无条件稳定的全离散逼近格式. 然后, 导出 $L^{2}$ 模意义下的收玫结果和 $H^{1}$ 模意义下的超逼近结果 $O\left(h^{2}+\tau^{\min \left\{2-\alpha_{1}, 3-\alpha\right\}}\right)$, 其中 $h$ 和 $\tau$ 分别表示空间和时间步长. 最后, 利用插值后处理技巧, 得到两项时间混合分数阶扩散波动方程有 限元逼近格式的整体超收玫结果. 本文的创新之处在于: 不仅利用有限元方法对两项时间混合分数阶 扩散波动方程进行稳定性和收玫性分析, 而且还得到了超收玫结果, 在不大幅增加计算量的前提下, 提 高了数值逼近精度.

本文其余内容安排如下: 第 2 节基于双线性有限元方法和 L1-CN 格式, 构造全离散逼近格式. 第 3 节给出一些用于进行稳定性分析和误差估计的重要引理. 第 4 节证明逼近格式的无条件稳定性, 并 导出 $L^{2}$ 模意义下的收玫结果, $H^{1}$ 模意义下的超逼近结果以及超收玫结果 $O\left(h^{2}+\tau^{\min \left\{2-\alpha_{1}, 3-\alpha\right\}}\right)$. 第 5 节从数值模拟的角度进一步展示理论分析的正确性和高效性. 最后一节对本文进行简要的总结, 同 时讨论了其他几类有限单元用于两项时间混合分数阶扩散波动方程时的数值分析情况以及对近期相 关研究工作的展望.

\section{2 有限元方法全离散逼近格式}

不妨令 $\Omega$ 为矩形区域, $\Gamma_{h}$ 为 $\Omega$ 的一族矩形网格剖分, 其中 $\bar{\Omega}=\cup_{K \in \Gamma_{h}} K$. 对于每一个 $K \in \Gamma_{h}$, 假设 $O_{K}$ 是 $K$ 的中心, 其中 $O_{K}=\left(x_{K}, y_{K}\right), h_{x, K}$, 和 $h_{y, K}$ 分别是 $O_{K}$ 到 $K$ 的平行于纵横坐标轴的 
两边的垂直距离. $K$ 的 4 个顶点分别为 $d_{1}=\left(x_{K}-h_{x, K}, y_{K}-h_{y, K}\right), d_{2}=\left(x_{K}+h_{x, K}, y_{K}-h_{y, K}\right), d_{3}=$ $\left(x_{K}+h_{x, K}, y_{K}+h_{y, K}\right), d_{4}=\left(x_{K}-h_{x, K}, y_{K}+h_{y, K}\right)$. 我们假设 $h_{K}=\max \left\{h_{x, K}, h_{y, K}\right\}, h=\max _{K \in \Gamma_{h}} h_{K}$, 定义有限元空间为

$$
V^{h}=\left\{v ;\left.v\right|_{K} \in Q_{11}(K)\right\}, V_{0}^{h}=\left\{v \in V^{h} ;\left.v\right|_{\partial \Omega}=0\right\},
$$

其中 $Q_{11}(K)=\operatorname{span}\left\{x^{r} y^{s} \mid(x, y) \in K, 0 \leqslant r \leqslant 1,0 \leqslant s \leqslant 1\right\}$.

设 $I_{h}: v \in H^{2}(\Omega) \rightarrow I_{h} v \in V^{h}$ 为一个插值算子, 且满足

$$
\left.I_{h}\right|_{K}=I_{K}, \quad I_{K} v\left(d_{i}\right)=v\left(d_{i}\right), \quad i=1,2,3,4 .
$$

则 (1) 式相应的弱形式为: 求 $u(\boldsymbol{x}, t):(0, T] \rightarrow H_{0}^{1}(\Omega)$, 使得

$$
\begin{cases}\left(D_{t}^{\alpha_{1}} u(\boldsymbol{x}, t), v\right)+\left(D_{t}^{\alpha} u(\boldsymbol{x}, t), v\right)+(\nabla u(\boldsymbol{x}, t), \nabla v)=(f(\boldsymbol{x}, t), v), & \forall v \in H_{0}^{1}(\Omega), \\ u(\boldsymbol{x}, 0)=u_{0}(\boldsymbol{x}), u_{t}(\boldsymbol{x}, 0)=\tilde{u}_{0}(\boldsymbol{x}), & \boldsymbol{x} \in \Omega .\end{cases}
$$

记 $(\cdot, \cdot)$ 和 $\|\cdot\|_{0}$ 分别为空间 $L^{2}(\Omega)$ 上定义的内积与 $L^{2}$ 模, 且 $\|\cdot\|_{m}$ 为空间 $H^{m}(\Omega)$ 中的模.

令 $0=t_{0}<t_{1}<\cdots<t_{N}=T$, 则相应的时间步长 $\tau=T / N$ 和 $t_{n}=n \tau, n=0,1, \ldots, N$. 对于区间 $[0, T]$ 上的光滑函数 $\varphi(t)$, 我们定义

$$
\begin{aligned}
& \varphi^{n}=\varphi\left(t_{n}\right), \quad \varphi(\boldsymbol{x}, t)=u_{t}(\boldsymbol{x}, t), \quad \varphi^{0}(\boldsymbol{x})=u_{t}(\boldsymbol{x}, 0)=\tilde{u}_{0}, \\
& \varphi^{n-\frac{1}{2}}=\frac{\varphi^{n}+\varphi^{n-1}}{2}, \quad \partial_{t} \varphi^{k-\frac{1}{2}}=\frac{\varphi^{k}-\varphi^{k-1}}{\tau}(1 \leqslant k \leqslant N), \quad \partial_{t} \varphi^{0}=0 \\
& \tilde{D}_{t}^{\alpha_{1}} u^{n-\frac{1}{2}}=\frac{\tau^{1-\alpha_{1}}}{2 \Gamma\left(2-\alpha_{1}\right)}\left(\sum_{k=1}^{n} \tilde{b}_{\alpha_{1}, n-k} \partial_{t} u^{k-\frac{1}{2}}+\sum_{k=1}^{n-1} \tilde{b}_{\alpha_{1}, n-k-1} \partial_{t} u^{k-\frac{1}{2}}\right) \\
& \tilde{D}_{t}^{\alpha} u^{n-\frac{1}{2}}=\frac{\tau^{1-\alpha}}{\Gamma(3-\alpha)}\left[\tilde{b}_{\alpha, 0} \partial_{t} u^{n-\frac{1}{2}}+\sum_{k=1}^{n-1}\left(\tilde{b}_{\alpha, n-k}-\tilde{b}_{\alpha, n-k-1}\right) \partial_{t} u^{k-\frac{1}{2}}-\tilde{b}_{\alpha, n-1} \tilde{u}_{0}\right]
\end{aligned}
$$

其中 $\tilde{b}_{\alpha_{1}, k}=(k+1)^{1-\alpha_{1}}-k^{1-\alpha_{1}}, \quad \tilde{b}_{\alpha, k}=(k+1)^{2-\alpha}-k^{2-\alpha}, 0 \leqslant k \leqslant N-1$.

基于 L1-CN 格式和 (2) 式, 可得如下全离散格式: 求 $U^{n} \in V_{0}^{h}$, 使得

$$
\left\{\begin{array}{lc}
\left(\tilde{D}_{t}^{\alpha_{1}} U^{n-\frac{1}{2}}, v^{h}\right)+\left(\tilde{D}_{t}^{\alpha} U^{n-\frac{1}{2}}, v^{h}\right)+\left(\nabla U^{n-\frac{1}{2}}, \nabla v^{h}\right)=\left(f^{n-\frac{1}{2}}, v^{h}\right), & \forall v^{h} \in V_{0}^{h}, \\
U^{0}=I_{h} u_{0}(\boldsymbol{x}), \quad \tilde{U}_{0}=I_{h} \tilde{u_{0}}, & \boldsymbol{x} \in \Omega .
\end{array}\right.
$$

\section{3 一些重要引理}

本节将给出用于稳定性分析和误差估计的一些重要引理.

引理1 $([39,40])$ 若 $u \in H^{4}(\Omega)$, 则

$$
\left(\nabla\left(u-I_{h} u\right), \nabla v^{h}\right)=O\left(h^{2}\right)|u|_{4}\left\|v^{h}\right\|_{0}, \quad \forall v^{h} \in V_{0}^{h} .
$$


引理2 ([41]) (1) 对 $\forall t \in(0, T]$, 若 $u_{t t}(\boldsymbol{x}, t) \in L^{2}(\Omega)$, 记

$$
R_{\alpha_{1}}^{n-\frac{1}{2}}=D_{t}^{\alpha_{1}} u^{n-\frac{1}{2}}-\tilde{D}_{t}^{\alpha_{1}} u^{n-\frac{1}{2}},
$$

则有

$$
\left\|R_{\alpha_{1}}^{n-\frac{1}{2}}\right\|_{0} \leqslant C \max _{0 \leqslant t \leqslant T}\left\|u_{t t}(\boldsymbol{x}, t)\right\|_{0} \tau^{2-\alpha_{1}} .
$$

(2) 若 $u_{t t t}(\boldsymbol{x}, t) \in L^{2}(\Omega)$, 记

$$
R_{\alpha}^{n-\frac{1}{2}}=D_{t}^{\alpha} u^{n-\frac{1}{2}}-\tilde{D}_{t}^{\alpha} u^{n-\frac{1}{2}}
$$

则有

$$
\left\|R_{\alpha}^{n-\frac{1}{2}}\right\|_{0} \leqslant C \max _{0 \leqslant t \leqslant T}\left\|u_{t t t}(\boldsymbol{x}, t)\right\|_{0} \tau^{3-\alpha} .
$$

本文中的 $C$ 是一个与 $h$ 和 $\tau$ 无关的正的常数, 它在不同地方可代表不同值.

引理3 ([42]) 设 $\left\{a_{0}, a_{1}, \ldots, a_{n}, \ldots\right\}$ 是一个实数序列且具有以下性质:

$$
a_{n} \geqslant 0, \quad a_{n}-a_{n-1} \leqslant 0, \quad a_{n+1}-2 a_{n}+a_{n-1} \geqslant 0,
$$

则对于任意的正整数 $M$ 和每一个由 $M$ 个实数组成的矢量 $\left(V_{1}, V_{2}, \ldots, V_{M}\right)$, 有

$$
\sum_{n=1}^{M}\left(\sum_{p=0}^{n-1} a_{p} V_{n-p}\right) V_{n} \geqslant 0
$$

引理4 ([43,44]) 对本文中的序列 $\tilde{b}_{\alpha, k}, k=0,1,2, \ldots$, 则有

$$
\begin{gathered}
1=\tilde{b}_{\alpha, 0}>\tilde{b}_{\alpha, 1}>\tilde{b}_{\alpha, 2}>\cdots>\tilde{b}_{\alpha, k}>\cdots>0, \\
(2-\alpha)(k+1)^{1-\alpha}<\tilde{b}_{\alpha, k}<(2-\alpha) k^{1-\alpha} .
\end{gathered}
$$

引理5 ([45]) 对本文中的序列 $\tilde{b}_{\alpha_{1}, k}=(k+1)^{1-\alpha_{1}}-k^{1-\alpha_{1}}, k=0,1,2, \ldots, n$, 对于任意的正整数 $N$ 和矢量 $\boldsymbol{Q}=\left[v^{1}, v^{2}, v^{3}, \ldots, v^{N-1}, v^{N}\right] \in \mathbb{R}^{N}$, 则有

$$
\sum_{n=1}^{N} \sum_{k=1}^{n} \tilde{b}_{\alpha_{1}, n-k} v^{k} v^{n} \geqslant 0, \quad \sum_{n=1}^{N} \sum_{k=1}^{n} \tilde{b}_{\alpha_{1}, n-k} v^{k} v^{n}+\sum_{n=1}^{N} \sum_{k=1}^{n-1} \tilde{b}_{\alpha_{1}, n-k-1} v^{k} v^{n} \geqslant 0 .
$$

\section{4 全离散逼近格式的稳定性、收敛性、超逼近和超收敛分析}

本节首先对全离散格式的稳定性进行分析, 然后证明了其收玫性和超逼近性. 最后, 借助于插值 后处理技巧得到了超收敛结果.

定理1 设 $\left\{U^{n}\right\}$ 为 (3) 式的解, 则有

$$
\left\|U^{n}\right\|_{1} \leqslant C\left(\left\|\nabla U^{0}\right\|_{0}^{2}+C_{1}\left\|\tilde{U}_{0}\right\|_{0}^{2}+C_{2} \max _{0 \leqslant t \leqslant T}\|f(t)\|_{0}^{2}\right)^{\frac{1}{2}} .
$$


证明 为了得到稳定性结果, 不妨在 (3) 式中令 $v^{h}=\partial_{t} U^{n-\frac{1}{2}}$, 则可得到以下等式:

$$
\left(\tilde{D}_{t}^{\alpha_{1}} U^{n-\frac{1}{2}}, \partial_{t} U^{n-\frac{1}{2}}\right)+\left(\tilde{D}_{t}^{\alpha} U^{n-\frac{1}{2}}, \partial_{t} U^{n-\frac{1}{2}}\right)+\left(\nabla U^{n-\frac{1}{2}}, \nabla \partial_{t} U^{n-\frac{1}{2}}\right)=\left(f^{n-\frac{1}{2}}, \partial_{t} U^{n-\frac{1}{2}}\right) .
$$

由 $\tilde{D}_{t}^{\alpha_{1}} U^{n-\frac{1}{2}}, \tilde{D}_{t}^{\alpha} U^{n-\frac{1}{2}}$ 及 $\partial_{t} U^{n-\frac{1}{2}}$ 的定义可知, (7) 式左端的 3 项可以转化为

$$
\begin{aligned}
& \left(\tilde{D}_{t}^{\alpha_{1}} U^{n-\frac{1}{2}}, \partial_{t} U^{n-\frac{1}{2}}\right) \\
& \quad=\frac{\tau^{1-\alpha_{1}}}{2 \Gamma\left(2-\alpha_{1}\right)}\left[\left(\sum_{k=1}^{n} \tilde{b}_{\alpha_{1}, n-k} \partial_{t} U^{k-\frac{1}{2}}, \partial_{t} U^{n-\frac{1}{2}}\right)+\left(\sum_{k=1}^{n-1} \tilde{b}_{\alpha_{1}, n-k-1} \partial_{t} U^{k-\frac{1}{2}}, \partial_{t} U^{n-\frac{1}{2}}\right)\right],
\end{aligned}
$$

$$
\begin{gathered}
\left(\tilde{D}_{t}^{\alpha} U^{n-\frac{1}{2}}, \partial_{t} U^{n-\frac{1}{2}}\right) \\
=\frac{\tau^{1-\alpha}}{\Gamma(3-\alpha)}\left(\tilde{b}_{\alpha, 0} \partial_{t} U^{n-\frac{1}{2}}+\sum_{k=1}^{n-1}\left(\tilde{b}_{\alpha, n-k}-\tilde{b}_{\alpha, n-k-1}\right) \partial_{t} U^{k-\frac{1}{2}}-\tilde{b}_{\alpha, n-1} \tilde{U}_{0}, \partial_{t} U^{n-\frac{1}{2}}\right) \\
=\frac{\tau^{1-\alpha}}{\Gamma(3-\alpha)}\left[\left\|\partial_{t} U^{n-\frac{1}{2}}\right\|_{0}^{2}-\sum_{k=1}^{n-1}\left(\tilde{b}_{\alpha, n-k-1}-\tilde{b}_{\alpha, n-k}\right)\left(\partial_{t} U^{k-\frac{1}{2}}, \partial_{t} U^{n-\frac{1}{2}}\right)-\left(\tilde{b}_{\alpha, n-1} \tilde{U}_{0}, \partial_{t} U^{n-\frac{1}{2}}\right)\right] \\
\left(\nabla U^{n-\frac{1}{2}}, \nabla \partial_{t} U^{n-\frac{1}{2}}\right)=\frac{1}{2 \tau}\left(\left\|\nabla U^{n}\right\|_{0}^{2}-\left\|\nabla U^{n-1}\right\|_{0}^{2}\right) .
\end{gathered}
$$

接下来, 为了方便起见, 记 $\lambda=\frac{\tau^{1-\alpha}}{\Gamma(3-\alpha)}, \lambda_{1}=\frac{\tau^{1-\alpha_{1}}}{\Gamma\left(2-\alpha_{1}\right)}$, 将 (8) (10) 式代入到 (7) 式中, 并让 (7) 式 两端同乘 $2 \tau$, 可得到如下:

$$
\begin{aligned}
2 \lambda \tau & \left\|\partial_{t} U^{n-\frac{1}{2}}\right\|_{0}^{2}+\left\|\nabla U^{n}\right\|_{0}^{2}-\left\|\nabla U^{n-1}\right\|_{0}^{2} \\
= & 2 \lambda \tau \sum_{k=1}^{n-1}\left(\tilde{b}_{\alpha, n-k-1}-\tilde{b}_{\alpha, n-k}\right)\left(\partial_{t} U^{k-\frac{1}{2}}, \partial_{t} U^{n-\frac{1}{2}}\right)+2 \lambda \tau\left(\tilde{b}_{\alpha, n-1} \tilde{U}_{0}, \partial_{t} U^{n-\frac{1}{2}}\right) \\
& +2 \tau\left(f^{n-\frac{1}{2}}, \partial_{t} U^{n-\frac{1}{2}}\right)-\lambda_{1} \tau\left[\sum_{k=1}^{n}\left(\tilde{b}_{\alpha_{1}, n-k} \partial_{t} U^{k-\frac{1}{2}}, \partial_{t} U^{n-\frac{1}{2}}\right)+\sum_{k=1}^{n-1}\left(\tilde{b}_{\alpha_{1}, n-k-1} \partial_{t} U^{k-\frac{1}{2}}, \partial_{t} U^{n-\frac{1}{2}}\right)\right] \\
\leqslant & \lambda \tau \sum_{k=1}^{n-1}\left(\tilde{b}_{\alpha, n-k-1}-\tilde{b}_{\alpha, n-k}\right)\left(\left\|\partial_{t} U^{k-\frac{1}{2}}\right\|_{0}^{2}+\left\|\partial_{t} U^{n-\frac{1}{2}}\right\|_{0}^{2}\right)+\lambda \tau \tilde{b}_{\alpha, n-1}\left(\left\|\tilde{U}_{0}\right\|_{0}^{2}+\left\|\partial_{t} U^{n-\frac{1}{2}}\right\|_{0}^{2}\right) \\
& +2 \tau\left|\left(f^{n-\frac{1}{2}}, \partial_{t} U^{n-\frac{1}{2}}\right)\right|-\lambda_{1} \tau\left[\sum_{k=1}^{n}\left(\tilde{b}_{\alpha_{1}, n-k} \partial_{t} U^{k-\frac{1}{2}}, \partial_{t} U^{n-\frac{1}{2}}\right)+\sum_{k=1}^{n-1}\left(\tilde{b}_{\alpha_{1}, n-k-1} \partial_{t} U^{k-\frac{1}{2}}, \partial_{t} U^{n-\frac{1}{2}}\right)\right] .
\end{aligned}
$$

令 $\rho^{0}=\left\|\nabla U^{0}\right\|_{0}^{2}$ 和 $\rho^{n}=\left\|\nabla U^{n}\right\|_{0}^{2}+\lambda \tau \sum_{k=1}^{n} \tilde{b}_{\alpha, n-k}\left\|\partial_{t} U^{k-\frac{1}{2}}\right\|_{0}^{2}$, 则 (11) 式可变形为

$$
\begin{aligned}
\rho^{n} \leqslant & \rho^{n-1}+\lambda \tau \tilde{b}_{\alpha, n-1}\left\|\tilde{U}_{0}\right\|_{0}^{2}+2 \tau\left|\left(f^{n-\frac{1}{2}}, \partial_{t} U^{n-\frac{1}{2}}\right)\right| \\
& -\lambda_{1} \tau\left[\sum_{k=1}^{n}\left(\tilde{b}_{\alpha_{1}, n-k} \partial_{t} U^{k-\frac{1}{2}}, \partial_{t} U^{n-\frac{1}{2}}\right)+\sum_{k=1}^{n-1}\left(\tilde{b}_{\alpha_{1}, n-k-1} \partial_{t} U^{k-\frac{1}{2}}, \partial_{t} U^{n-\frac{1}{2}}\right)\right] \\
\leqslant & \rho^{n-2}+\lambda \tau \tilde{b}_{\alpha, n-1}\left\|\tilde{U}_{0}\right\|_{0}^{2}+2 \tau\left|\left(f^{n-\frac{1}{2}}, \partial_{t} U^{n-\frac{1}{2}}\right)\right| \\
& -\lambda_{1} \tau\left[\sum_{k=1}^{n}\left(\tilde{b}_{\alpha_{1}, n-k} \partial_{t} U^{k-\frac{1}{2}}, \partial_{t} U^{n-\frac{1}{2}}\right)+\sum_{k=1}^{n-1}\left(\tilde{b}_{\alpha_{1}, n-k-1} \partial_{t} U^{k-\frac{1}{2}}, \partial_{t} U^{n-\frac{1}{2}}\right)\right]
\end{aligned}
$$




$$
\begin{aligned}
& \quad+\lambda \tau \tilde{b}_{\alpha, n-2}\left\|\tilde{U}_{0}\right\|_{0}^{2}+2 \tau \mid\left(f^{n-1-\frac{1}{2}}, \partial_{t} U^{n-1-\frac{1}{2}}\right) \\
& \quad-\lambda_{1} \tau\left[\sum_{k=1}^{n}\left(\tilde{b}_{\alpha_{1}, n-k-1} \partial_{t} U^{k-\frac{1}{2}}, \partial_{t} U^{n-1-\frac{1}{2}}\right)+\sum_{k=1}^{n-1}\left(\tilde{b}_{\alpha_{1}, n-k-2} \partial_{t} U^{k-\frac{1}{2}}, \partial_{t} U^{n-1-\frac{1}{2}}\right)\right] \\
& \leqslant \cdots
\end{aligned}
$$

对以上式子, 用 $l$ 代替 $n$, 并对 $l$ 从 1 到 $n$ 求和, 然后利用 Cauchy-Schwarz 不等式和 Young's 不 等式, 则可得以下估计:

$$
\begin{aligned}
\rho^{n} \leqslant & \rho^{0}+\lambda \tau \sum_{l=1}^{n} \tilde{b}_{\alpha, l-1}\left\|\tilde{U}_{0}\right\|_{0}^{2}+2 \tau \sum_{l=1}^{n}\left|\left(f^{l-\frac{1}{2}}, \partial_{t} U^{l-\frac{1}{2}}\right)\right| \\
& -\lambda_{1} \tau \sum_{l=1}^{n}\left[\sum_{k=1}^{l}\left(\tilde{b}_{\alpha_{1}, l-k} \partial_{t} U^{k-\frac{1}{2}}, \partial_{t} U^{l-\frac{1}{2}}\right)+\sum_{k=1}^{l-1}\left(\tilde{b}_{\alpha_{1}, l-k-1} \partial_{t} U^{k-\frac{1}{2}}, \partial_{t} U^{l-\frac{1}{2}}\right)\right] \\
\leqslant & \rho^{0}+\lambda \tau \sum_{l=1}^{n} \tilde{b}_{\alpha, l-1}\left\|\tilde{U}_{0}\right\|_{0}^{2}+\sum_{l=1}^{n} \frac{\tau\left\|f^{l-\frac{1}{2}}\right\|_{0}^{2}}{\lambda \tilde{b}_{\alpha, n-l}}+\sum_{l=1}^{n} \lambda \tau \tilde{b}_{\alpha, n-l}\left\|\partial_{t} U^{l-\frac{1}{2}}\right\|_{0}^{2} \\
& -\lambda_{1} \tau \sum_{l=1}^{n}\left[\sum_{k=1}^{l}\left(\tilde{b}_{\alpha_{1}, l-k} \partial_{t} U^{k-\frac{1}{2}}, \partial_{t} U^{l-\frac{1}{2}}\right)+\sum_{k=1}^{l-1}\left(\tilde{b}_{\alpha_{1}, l-k-1} \partial_{t} U^{k-\frac{1}{2}}, \partial_{t} U^{l-\frac{1}{2}}\right)\right] .
\end{aligned}
$$

即

$$
\begin{aligned}
\left\|\nabla U^{n}\right\|_{0}^{2} \leqslant & \left\|\nabla U^{0}\right\|_{0}^{2}+\lambda \tau \sum_{l=1}^{n} \tilde{b}_{\alpha, l-1}\left\|\tilde{U}_{0}\right\|_{0}^{2}+\sum_{l=1}^{n} \frac{\tau\left\|f^{l-\frac{1}{2}}\right\|_{0}^{2}}{\lambda \tilde{b}_{\alpha, n-l}} \\
& -\lambda_{1} \tau \sum_{l=1}^{n}\left[\sum_{k=1}^{l}\left(\tilde{b}_{\alpha_{1}, l-k} \partial_{t} U^{k-\frac{1}{2}}, \partial_{t} U^{l-\frac{1}{2}}\right)+\sum_{k=1}^{l-1}\left(\tilde{b}_{\alpha_{1}, l-k-1} \partial_{t} U^{k-\frac{1}{2}}, \partial_{t} U^{l-\frac{1}{2}}\right)\right] .
\end{aligned}
$$

再利用引理 4 , 可得

$$
\lambda \tau \sum_{l=1}^{n} \tilde{b}_{\alpha, l-1}\left\|\tilde{U}_{0}\right\|_{0}^{2} \leqslant \frac{T^{2-\alpha}}{\Gamma(3-\alpha)}\left\|\tilde{U}_{0}\right\|_{0}^{2}, \quad \sum_{l=1}^{n} \frac{\tau\left\|f^{l-\frac{1}{2}}\right\|_{0}^{2}}{\lambda \tilde{b}_{\alpha, n-l}} \leqslant \frac{\Gamma(3-\alpha) T^{\alpha}}{2-\alpha} \max _{0 \leqslant t \leqslant T}\|f(\boldsymbol{x}, t)\|_{0}^{2} .
$$

此外, 根据引理 5 , 可以推断

$$
-\lambda_{1} \tau \sum_{l=1}^{n}\left[\sum_{k=1}^{l}\left(\tilde{b}_{\alpha_{1}, l-k} \partial_{t} U^{k-\frac{1}{2}}, \partial_{t} U^{l-\frac{1}{2}}\right)+\sum_{k=1}^{l-1}\left(\tilde{b}_{\alpha_{1}, l-k-1} \partial_{t} U^{k-\frac{1}{2}}, \partial_{t} U^{l-\frac{1}{2}}\right)\right] \leqslant 0 .
$$

然后将以上 (15) 和 (16) 式代入到 (14) 式, 则有

$$
\left\|\nabla U^{n}\right\|_{0}^{2} \leqslant\left\|\nabla U^{0}\right\|_{0}^{2}+\frac{T^{2-\alpha}}{\Gamma(3-\alpha)}\left\|\tilde{U}_{0}\right\|_{0}^{2}+\frac{\Gamma(3-\alpha) T^{\alpha}}{2-\alpha} \max _{0 \leqslant t \leqslant T}\|f(\boldsymbol{x}, t)\|_{0}^{2},
$$

即

$$
\left\|\nabla U^{n}\right\|_{0}^{2} \leqslant\left\|\nabla U^{0}\right\|_{0}^{2}+C_{1}\left\|\tilde{U}_{0}\right\|_{0}^{2}+C_{2} \max _{0 \leqslant t \leqslant T}\|f(\boldsymbol{x}, t)\|_{0}^{2}
$$

其中 $C_{1}=\frac{T^{2-\alpha}}{\Gamma(3-\alpha)}, C_{2}=\frac{\Gamma(3-\alpha) T^{\alpha}}{2-\alpha}$.

因 $U^{n} \in H_{0}^{1}(\Omega)$, 且在空间 $H_{0}^{1}(\Omega)$ 中, 由 $H^{1}$ 模和半模之间的等价性, 易得定理结果.

接下来, 借助于定理 2 , 给出有限元全离散逼近格式的收玫结果和超逼近结果. 
定理 2 设 $u^{n}$ 是 (2) 式在 $t=t_{n}$ 时的解, $U^{n}$ 为 (3) 式的解, 且 $u \in H^{4}(\Omega), u_{t} \in H^{2}(\Omega), u_{t t} \in$ $L^{2}(\Omega), u_{t t t} \in L^{2}(\Omega)$, 则对任意的整数 $1 \leqslant n \leqslant N$, 有

$$
\left\|u^{n}-U^{n}\right\|_{0}=O\left(h^{2}+\tau^{\min \left\{2-\alpha_{1}, 3-\alpha\right\}}\right), \quad\left\|I_{h} u^{n}-U^{n}\right\|_{1}=O\left(h^{2}+\tau^{\min \left\{2-\alpha_{1}, 3-\alpha\right\}}\right) .
$$

证明 由 (2) 和 (3) 式, 可以得到以下误差方程:

$$
\begin{aligned}
& \left(\tilde{D}_{t}^{\alpha_{1}}\left(u^{n-\frac{1}{2}}-U^{n-\frac{1}{2}}\right), v^{h}\right)+\left(\tilde{D}_{t}^{\alpha}\left(u^{n-\frac{1}{2}}-U^{n-\frac{1}{2}}\right), v^{h}\right)+\left(\nabla\left(u^{n-\frac{1}{2}}-U^{n-\frac{1}{2}}\right), \nabla v^{h}\right) \\
& \quad=-\left(R_{\alpha_{1}}^{n-\frac{1}{2}}, v^{h}\right)-\left(R_{\alpha}^{n-\frac{1}{2}}, v^{h}\right), \quad \forall v^{h} \in V_{0}^{h} .
\end{aligned}
$$

记 $\eta^{n}=u^{n}-I_{h} u^{n}, \xi^{n}=I_{h} u^{n}-U^{n}$, 并在 (17) 式中令 $v^{h}=\partial_{t} \xi^{n-\frac{1}{2}}$, 则有

$$
\begin{aligned}
\left(\tilde{D}_{t}^{\alpha_{1}} \xi^{n-\frac{1}{2}}, \partial_{t} \xi^{n-\frac{1}{2}}\right)+\left(\tilde{D}_{t}^{\alpha} \xi^{n-\frac{1}{2}}, \partial_{t} \xi^{n-\frac{1}{2}}\right)+\left(\nabla \xi^{n-\frac{1}{2}}, \nabla \partial_{t} \xi^{n-\frac{1}{2}}\right) & \\
= & -\left(\tilde{D}_{t}^{\alpha_{1}} \eta^{n-\frac{1}{2}}, \partial_{t} \xi^{n-\frac{1}{2}}\right)-\left(\tilde{D}_{t}^{\alpha} \eta^{n-\frac{1}{2}}, \partial_{t} \xi^{n-\frac{1}{2}}\right)-\left(\nabla \eta^{n-\frac{1}{2}}, \nabla \partial_{t} \xi^{n-\frac{1}{2}}\right) \\
& -\left(R_{\alpha_{1}}^{n-\frac{1}{2}}, \partial_{t} \xi^{n-\frac{1}{2}}\right)-\left(R_{\alpha}^{n-\frac{1}{2}}, \partial_{t} \xi^{n-\frac{1}{2}}\right) .
\end{aligned}
$$

由 $\tilde{D}_{t}^{\alpha_{1}} U^{n-\frac{1}{2}}, \tilde{D}_{t}^{\alpha} U^{n-\frac{1}{2}}$ 及 $\partial_{t} \xi^{n-\frac{1}{2}}$ 的定义可知, (18) 式的左端 3 项可化为

$$
\begin{aligned}
& \left(\tilde{D}_{t}^{\alpha_{1}} \xi^{n-\frac{1}{2}}, \partial_{t} \xi^{n-\frac{1}{2}}\right) \\
& \quad=\frac{\tau^{1-\alpha_{1}}}{2 \Gamma\left(2-\alpha_{1}\right)}\left[\left(\sum_{k=1}^{n} \tilde{b}_{\alpha_{1}, n-k} \partial_{t} \xi^{k-\frac{1}{2}}, \partial_{t} \xi^{n-\frac{1}{2}}\right)+\left(\sum_{k=1}^{n-1} \tilde{b}_{\alpha_{1}, n-k-1} \partial_{t} \xi^{k-\frac{1}{2}}, \partial_{t} \xi^{n-\frac{1}{2}}\right)\right],
\end{aligned}
$$

$$
\begin{gathered}
\left(\tilde{D}_{t}^{\alpha} \xi^{n-\frac{1}{2}}, \partial_{t} \xi^{n-\frac{1}{2}}\right) \\
=\frac{\tau^{1-\alpha}}{\Gamma(3-\alpha)}\left(\tilde{b}_{\alpha, 0} \partial_{t} \xi^{n-\frac{1}{2}}+\sum_{k=1}^{n-1}\left(\tilde{b}_{\alpha, n-k}-\tilde{b}_{\alpha, n-k-1}\right) \partial_{t} \xi^{k-\frac{1}{2}}-\tilde{b}_{\alpha, n-1} \tilde{\xi}_{0}, \partial_{t} \xi^{n-\frac{1}{2}}\right) \\
=\frac{\tau^{1-\alpha}}{\Gamma(3-\alpha)}\left[\left\|\partial_{t} \xi^{n-\frac{1}{2}}\right\|_{0}^{2}-\sum_{k=1}^{n-1}\left(\tilde{b}_{\alpha, n-k-1}-\tilde{b}_{\alpha, n-k}\right)\left(\partial_{t} \xi^{k-\frac{1}{2}}, \partial_{t} \xi^{n-\frac{1}{2}}\right)-\left(\tilde{b}_{\alpha, n-1} \tilde{\xi}_{0}, \partial_{t} \xi^{n-\frac{1}{2}}\right)\right], \\
\left(\nabla \xi^{n-\frac{1}{2}}, \nabla \partial_{t} \xi^{n-\frac{1}{2}}\right)=\frac{1}{2 \tau}\left(\left\|\nabla \xi^{n}\right\|_{0}^{2}-\left\|\nabla \xi^{n-1}\right\|_{0}^{2}\right) .
\end{gathered}
$$

接下来, 令 $\lambda=\frac{\tau^{1-\alpha}}{\Gamma(3-\alpha)}, \lambda_{1}=\frac{\tau^{1-\alpha_{1}}}{\Gamma\left(2-\alpha_{1}\right)}$, 然后将 (19) (21) 式代入到 (18) 式中, 并让 (18) 式两端同 乘 $2 \tau$, 可得到如下:

$$
\begin{aligned}
2 \lambda \tau & \left\|\partial_{t} \xi^{n-\frac{1}{2}}\right\|_{0}^{2}+\left\|\nabla \xi^{n}\right\|_{0}^{2}-\left\|\nabla \xi^{n-1}\right\|_{0}^{2} \\
= & 2 \lambda \tau \sum_{k=1}^{n-1}\left(\tilde{b}_{\alpha, n-k-1}-\tilde{b}_{\alpha, n-k}\right)\left(\partial_{t} \xi^{k-\frac{1}{2}}, \partial_{t} \xi^{n-\frac{1}{2}}\right)+2 \lambda \tau\left(\tilde{b}_{\alpha, n-1} \tilde{\xi}_{0}, \partial_{t} \xi^{n-\frac{1}{2}}\right) \\
& -2 \tau\left(\tilde{D}_{t}^{\alpha_{1}} \eta^{n-\frac{1}{2}}, \partial_{t} \xi^{n-\frac{1}{2}}\right)-2 \tau\left(\tilde{D}_{t}^{\alpha} \eta^{n-\frac{1}{2}}, \partial_{t} \xi^{n-\frac{1}{2}}\right)-2 \tau\left(\nabla \eta^{n-\frac{1}{2}}, \nabla \partial_{t} \xi^{n-\frac{1}{2}}\right)-2 \tau\left(R_{\alpha_{1}}^{n-\frac{1}{2}}, \partial_{t} \xi^{n-\frac{1}{2}}\right) \\
& -2 \tau\left(R_{\alpha}^{n-\frac{1}{2}}, \partial_{t} \xi^{n-\frac{1}{2}}\right)-\lambda_{1} \tau\left[\sum_{k=1}^{n}\left(\tilde{b}_{\alpha_{1}, n-k} \partial_{t} \xi^{k-\frac{1}{2}}, \partial_{t} \xi^{n-\frac{1}{2}}\right)+\sum_{k=1}^{n-1}\left(\tilde{b}_{\alpha_{1}, n-k-1} \partial_{t} \xi^{k-\frac{1}{2}}, \partial_{t} \xi^{n-\frac{1}{2}}\right)\right] \\
\leqslant & \lambda \tau \sum_{k=1}^{n-1}\left(\tilde{b}_{\alpha, n-k-1}-\tilde{b}_{\alpha, n-k}\right)\left(\left\|\partial_{t} \xi^{k-\frac{1}{2}}\right\|_{0}^{2}+\left\|\partial_{t} \xi^{n-\frac{1}{2}}\right\|_{0}^{2}\right)+\lambda \tau \tilde{b}_{\alpha, n-1}\left(\left\|\tilde{\xi}_{0}\right\|_{0}^{2}+\left\|\partial_{t} \xi^{n-\frac{1}{2}}\right\|_{0}^{2}\right)
\end{aligned}
$$




$$
\begin{aligned}
& -2 \tau\left(\tilde{D}_{t}^{\alpha_{1}} \eta^{n-\frac{1}{2}}, \partial_{t} \xi^{n-\frac{1}{2}}\right)-2 \tau\left(\tilde{D}_{t}^{\alpha} \eta^{n-\frac{1}{2}}, \partial_{t} \xi^{n-\frac{1}{2}}\right)-2 \tau\left(\nabla \eta^{n-\frac{1}{2}}, \nabla \partial_{t} \xi^{n-\frac{1}{2}}\right)-2 \tau\left(R_{\alpha_{1}}^{n-\frac{1}{2}}, \partial_{t} \xi^{n-\frac{1}{2}}\right) \\
& -2 \tau\left(R_{\alpha}^{n-\frac{1}{2}}, \partial_{t} \xi^{n-\frac{1}{2}}\right)-\lambda_{1} \tau\left[\sum_{k=1}^{n}\left(\tilde{b}_{\alpha_{1}, n-k} \partial_{t} \xi^{k-\frac{1}{2}}, \partial_{t} \xi^{n-\frac{1}{2}}\right)+\sum_{k=1}^{n-1}\left(\tilde{b}_{\alpha_{1}, n-k-1} \partial_{t} \xi^{k-\frac{1}{2}}, \partial_{t} \xi^{n-\frac{1}{2}}\right)\right] .
\end{aligned}
$$

记 $\rho^{0}=\left\|\nabla \xi^{0}\right\|_{0}^{2}$ 和 $\rho^{n}=\left\|\nabla \xi^{n}\right\|_{0}^{2}+\lambda \tau \sum_{k=1}^{n} \tilde{b}_{\alpha, n-k}\left\|\partial_{t} \xi^{k-\frac{1}{2}}\right\|_{0}^{2}$, 那么 (22) 式就等价于

$$
\begin{aligned}
\rho^{n} \leqslant & \rho^{n-1}+\lambda \tau \tilde{b}_{\alpha, n-1}\left\|\tilde{\xi}_{0}\right\|_{0}^{2}-2 \tau\left(\tilde{D}_{t}^{\alpha_{1}} \eta^{n-\frac{1}{2}}, \partial_{t} \xi^{n-\frac{1}{2}}\right)-2 \tau\left(\tilde{D}_{t}^{\alpha} \eta^{n-\frac{1}{2}}, \partial_{t} \xi^{n-\frac{1}{2}}\right) \\
& -2 \tau\left(\nabla \eta^{n-\frac{1}{2}}, \nabla \partial_{t} \xi^{n-\frac{1}{2}}\right)-2 \tau\left(R_{\alpha_{1}}^{n-\frac{1}{2}}, \partial_{t} \xi^{n-\frac{1}{2}}\right)-2 \tau\left(R_{\alpha}^{n-\frac{1}{2}}, \partial_{t} \xi^{n-\frac{1}{2}}\right) \\
& -\lambda_{1} \tau\left[\sum_{k=1}^{n}\left(\tilde{b}_{\alpha_{1}, n-k} \partial_{t} \xi^{k-\frac{1}{2}}, \partial_{t} \xi^{n-\frac{1}{2}}\right)+\sum_{k=1}^{n-1}\left(\tilde{b}_{\alpha_{1}, n-k-1} \partial_{t} \xi^{k-\frac{1}{2}}, \partial_{t} \xi^{n-\frac{1}{2}}\right)\right] \\
\leqslant & \rho^{n-2}+\lambda \tau \tilde{b}_{\alpha, n-1}\left\|\tilde{\xi}_{0}\right\|_{0}^{2}-2 \tau\left(\tilde{D}_{t}^{\alpha_{1}} \eta^{n-\frac{1}{2}}, \partial_{t} \xi^{n-\frac{1}{2}}\right)-2 \tau\left(\tilde{D}_{t}^{\alpha} \eta^{n-\frac{1}{2}}, \partial_{t} \xi^{n-\frac{1}{2}}\right) \\
& -2 \tau\left(\nabla \eta^{n-\frac{1}{2}}, \nabla \partial_{t} \xi^{n-\frac{1}{2}}\right)-2 \tau\left(R_{\alpha_{1}}^{n-\frac{1}{2}}, \partial_{t} \xi^{n-\frac{1}{2}}\right)-2 \tau\left(R_{\alpha}^{n-\frac{1}{2}}, \partial_{t} \xi^{n-\frac{1}{2}}\right) \\
& -\lambda_{1} \tau\left[\sum_{k=1}^{n}\left(\tilde{b}_{\alpha_{1}, n-k} \partial_{t} \xi^{k-\frac{1}{2}}, \partial_{t} \xi^{n-\frac{1}{2}}\right)+\sum_{k=1}^{n-1}\left(\tilde{b}_{\alpha_{1}, n-k-1} \partial_{t} \xi^{k-\frac{1}{2}}, \partial_{t} \xi^{n-\frac{1}{2}}\right)\right] \\
& +\lambda \tau \tilde{b}_{\alpha, n-2}\left\|\tilde{\xi}_{0}\right\|_{0}^{2}-2 \tau\left(\tilde{D}_{t}^{\alpha_{1}} \eta^{n-1-\frac{1}{2}}, \partial_{t} \xi^{n-1-\frac{1}{2}}\right)-2 \tau\left(\tilde{D}_{t}^{\alpha} \eta^{n-1-\frac{1}{2}}, \partial_{t} \xi^{n-1-\frac{1}{2}}\right) \\
& -2 \tau\left(\nabla \eta^{n-1-\frac{1}{2}}, \nabla \partial_{t} \xi^{n-1-\frac{1}{2}}\right)-2 \tau\left(R_{\alpha_{1}}^{n-1-\frac{1}{2}}, \partial_{t} \xi^{n-1-\frac{1}{2}}\right)-2 \tau\left(R_{\alpha}^{n-1-\frac{1}{2}}, \partial_{t} \xi^{n-1-\frac{1}{2}}\right) \\
& -\lambda_{1} \tau\left[\sum_{k=1}^{n}\left(\tilde{b}_{\alpha_{1}, n-k-1} \partial_{t} \xi^{k-\frac{1}{2}}, \partial_{t} \xi^{n-1-\frac{1}{2}}\right)+\sum_{k=1}^{n-1}\left(\tilde{b}_{\alpha_{1}, n-k-2} \partial_{t} \xi^{k-\frac{1}{2}}, \partial_{t} \xi^{n-1-\frac{1}{2}}\right)\right] \\
\leqslant & \cdots
\end{aligned}
$$

对上式用 $l$ 代替 $n$, 并对 $l$ 从 1 到 $n$ 求和, 则有

$$
\begin{aligned}
\rho^{n} \leqslant & \rho^{0}+\lambda \tau \sum_{l=1}^{n} \tilde{b}_{\alpha, l-1}\left\|\tilde{\xi}_{0}\right\|_{0}^{2}-2 \tau \sum_{l=1}^{n}\left(\tilde{D}_{t}^{\alpha_{1}} \eta^{l-\frac{1}{2}}, \partial_{t} \xi^{l-\frac{1}{2}}\right)-2 \tau \sum_{l=1}^{n}\left(\tilde{D}_{t}^{\alpha} \eta^{l-\frac{1}{2}}, \partial_{t} \xi^{l-\frac{1}{2}}\right) \\
& -2 \tau \sum_{l=1}^{n}\left(\nabla \eta^{l-\frac{1}{2}}, \nabla \partial_{t} \xi^{l-\frac{1}{2}}\right)-2 \tau \sum_{l=1}^{n}\left(R_{\alpha_{1}}^{l-\frac{1}{2}}, \partial_{t} \xi^{l-\frac{1}{2}}\right)-2 \tau \sum_{l=1}^{n}\left(R_{\alpha}^{l-\frac{1}{2}}, \partial_{t} \xi^{l-\frac{1}{2}}\right) \\
& -\lambda_{1} \tau \sum_{l=1}^{n}\left[\sum_{k=1}^{l}\left(\tilde{b}_{\alpha_{1}, l-k} \partial_{t} \xi^{k-\frac{1}{2}}, \partial_{t} \xi^{l-\frac{1}{2}}\right)+\sum_{k=1}^{l-1}\left(\tilde{b}_{\alpha_{1}, l-k-1} \partial_{t} \xi^{k-\frac{1}{2}}, \partial_{t} \xi^{l-\frac{1}{2}}\right)\right]
\end{aligned}
$$

再利用 Cauchy-Schwarz 不等式和 Young's 不等式, 即得

$$
\begin{gathered}
2 \tau \sum_{l=1}^{n}\left(\tilde{D}_{t}^{\alpha_{1}} \eta^{l-\frac{1}{2}}, \partial_{t} \xi^{l-\frac{1}{2}}\right) \leqslant \sum_{l=1}^{n} \frac{5 \tau\left\|\tilde{D}_{t}^{\alpha_{1}} \eta^{l-\frac{1}{2}}\right\|_{0}^{2}}{\lambda \tilde{b}_{\alpha, n-l}}+\sum_{l=1}^{n} \frac{\lambda \tau \tilde{b}_{\alpha, n-l}\left\|\partial_{t} \xi^{l-\frac{1}{2}}\right\|_{0}^{2}}{5}, \\
2 \tau \sum_{l=1}^{n}\left(\tilde{D}_{t}^{\alpha} \eta^{l-\frac{1}{2}}, \partial_{t} \xi^{l-\frac{1}{2}}\right) \leqslant \sum_{l=1}^{n} \frac{5 \tau\left\|\tilde{D}_{t}^{\alpha} \eta^{l-\frac{1}{2}}\right\|_{0}^{2}}{\lambda \tilde{b}_{\alpha, n-l}}+\sum_{l=1}^{n} \frac{\lambda \tau \tilde{b}_{\alpha, n-l}\left\|\partial_{t} \xi^{l-\frac{1}{2}}\right\|_{0}^{2}}{5}, \\
2 \tau \sum_{l=1}^{n}\left(\nabla \eta^{l-\frac{1}{2}}, \nabla \partial_{t} \xi^{l-\frac{1}{2}}\right) \leqslant \sum_{l=1}^{n} \frac{C h^{4} \tau\|u\|_{L^{\infty}\left(H^{4}(\Omega)\right)}^{2}}{\lambda \tilde{b}_{\alpha, n-l}}+\sum_{l=1}^{n} \frac{\lambda \tau \tilde{b}_{\alpha, n-l}\left\|\partial_{t} \xi^{l-\frac{1}{2}}\right\|_{0}^{2}}{5}, \\
2 \tau \sum_{l=1}^{n}\left(R_{\alpha_{1}}^{l-\frac{1}{2}}, \partial_{t} \xi^{l-\frac{1}{2}}\right) \leqslant \frac{C \tau \max _{0 \leqslant t \leqslant T}\left\|u_{t t}(X, t)\right\|_{0}^{2} \tau^{4-2 \alpha_{1}}}{\lambda \tilde{b}_{\alpha, n-l}}+\sum_{l=1}^{n} \frac{\lambda \tau \tilde{b}_{\alpha, n-l}\left\|\partial_{t} \xi^{l-\frac{1}{2}}\right\|_{0}^{2}}{5}
\end{gathered}
$$




$$
2 \tau \sum_{l=1}^{n}\left(R_{\alpha}^{l-\frac{1}{2}}, \partial_{t} \xi^{l-\frac{1}{2}}\right) \leqslant \frac{C \tau \max _{0 \leqslant t \leqslant T}\left\|u_{t t t}(X, t)\right\|_{0}^{2} \tau^{6-2 \alpha}}{\lambda \tilde{b}_{\alpha, n-l}}+\sum_{l=1}^{n} \frac{\lambda \tau \tilde{b}_{\alpha, n-l}\left\|\partial_{t} \xi^{l-\frac{1}{2}}\right\|_{0}^{2}}{5} .
$$

由引理 4 可知

$$
\sum_{l=1}^{n} \frac{1}{\tilde{b}_{\alpha, n-l}} \leqslant \frac{n(k+1)^{\alpha-1}}{(2-\alpha)} \leqslant \frac{N^{\alpha}}{(2-\alpha)} \leqslant \frac{T^{\alpha} \tau^{-\alpha}}{2-\alpha} .
$$

再利用引理 5 , 可以推断

$$
-\lambda_{1} \tau \sum_{l=1}^{n}\left[\sum_{k=1}^{l}\left(\tilde{b}_{\alpha_{1}, l-k} \partial_{t} \xi^{k-\frac{1}{2}}, \partial_{t} \xi^{l-\frac{1}{2}}\right)+\sum_{k=1}^{l-1}\left(\tilde{b}_{\alpha_{1}, l-k-1} \partial_{t} \xi^{k-\frac{1}{2}}, \partial_{t} \xi^{l-\frac{1}{2}}\right)\right] \leqslant 0 .
$$

综上, 我们易得出以下各项估计:

$$
\begin{aligned}
& \sum_{l=1}^{n} \frac{5 \tau\left\|\tilde{D}_{t}^{\alpha_{1}} \eta^{l-\frac{1}{2}}\right\|_{0}^{2}}{\lambda \tilde{b}_{\alpha, n-l}} \leqslant \frac{5 T^{\alpha} \Gamma(3-\alpha)}{2-\alpha}\left\|\tilde{D}_{t}^{\alpha_{1}} \eta^{l-\frac{1}{2}}\right\|_{0}^{2} \leqslant C h^{4}\left\|\tilde{D}_{t}^{\alpha_{1}} u^{l-\frac{1}{2}}\right\|_{2}^{2}, \\
& \sum_{l=1}^{n} \frac{5 \tau\left\|\tilde{D}_{t}^{\alpha} \eta^{l-\frac{1}{2}}\right\|_{0}^{2}}{\lambda \tilde{b}_{\alpha, n-l}} \leqslant \frac{5 T^{\alpha} \Gamma(3-\alpha)}{2-\alpha}\left\|\tilde{D}_{t}^{\alpha_{1}} \eta^{l-\frac{1}{2}}\right\|_{0}^{2} \leqslant C h^{4}\left\|\tilde{D}_{t}^{\alpha_{1}} u^{l-\frac{1}{2}}\right\|_{2}^{2}, \\
& \sum_{l=1}^{n} \frac{C h^{4} \tau\|u\|_{L^{\infty}\left(H^{4}(\Omega)\right)}^{2}}{\lambda \tilde{b}_{\alpha, n-l}} \leqslant \frac{T^{\alpha} \Gamma(3-\alpha)}{2-\alpha} C h^{4}\|u\|_{L^{\infty}\left(H^{4}(\Omega)\right)}^{2}, \\
& \sum_{l=1}^{n} \frac{C \tau \max _{0 \leqslant t \leqslant T}\left\|u_{t t}(X, t)\right\|_{0}^{2} \tau^{4-2 \alpha_{1}}}{\lambda \tilde{b}_{\alpha, n-l}} \leqslant \frac{C T^{\alpha} \Gamma(3-\alpha)}{2-\alpha} \max _{0 \leqslant t \leqslant T}\left\|u_{t t}(X, t)\right\|_{0}^{2} \tau^{4-2 \alpha_{1}}, \\
& \sum_{l=1}^{n} \frac{C \tau \max _{0 \leqslant t \leqslant T}\left\|u_{t t t}(X, t)\right\|_{0}^{2} \tau^{6-2 \alpha}}{\lambda \tilde{b}_{\alpha, n-l}} \leqslant \frac{C T^{\alpha} \Gamma(3-\alpha)}{2-\alpha} \max _{0 \leqslant t \leqslant T}\left\|u_{t t t}(X, t)\right\|_{0}^{2} \tau^{6-2 \alpha} .
\end{aligned}
$$

综合 $(24) \sim(34)$ 式以及 $H_{0}^{1}(\Omega)$ 空间的性质可知

$$
\left\|I_{h} u^{n}-U^{n}\right\|_{1}=O\left(h^{2}+\tau^{\min \left\{2-\alpha_{1}, 3-\alpha\right\}}\right) .
$$

因此易得

$$
\left\|u^{n}-U^{n}\right\|_{0}=O\left(h^{2}+\tau^{\min \left\{2-\alpha_{1}, 3-\alpha\right\}}\right) .
$$

为了得到整体超收玫结果, 基于原剖分单元, 我们构造了一个新的剖分族, 记为 $\Gamma_{2 h}$, 新的单元 $\widetilde{K}$ 由 $\Gamma_{h}$ 中 4 个相邻的小单元构成.

如图 1, 根据文献 [39] 中的方法, 在 $\widetilde{K} \in \Gamma_{2 h}$ 上构造插值后处理算子 $I_{2 h}$, 记 $A_{i}(i=1, \ldots, 9)$ 为 4 个小单元的 9 个顶点. 对任意的 $t \in(0, T], I_{2 h}$ 满足

$$
\begin{gathered}
\left.I_{2 h} u\right|_{\widetilde{K}} \in Q_{22}(\widetilde{K}), \quad \forall u \in C(\widetilde{K}), \\
I_{2 h} u\left(A_{i}, t\right)=u\left(A_{i}, t\right), \quad i=1, \ldots, 9, \\
I_{2 h} I_{h} u=I_{2 h} u, \quad \forall u \in H^{3}(\Omega), \\
\left\|I_{2 h} u-u\right\|_{1} \leqslant C h^{2}\|u\|_{3}, \quad \forall u \in H^{3}(\Omega), \\
\left\|I_{2 h} v^{h}\right\|_{1} \leqslant C\left\|v^{h}\right\|_{1}, \quad \forall v^{h} \in V_{0}^{h} .
\end{gathered}
$$

由 $I_{2 h}$ 的性质, 我们可得以下超收玫结果. 


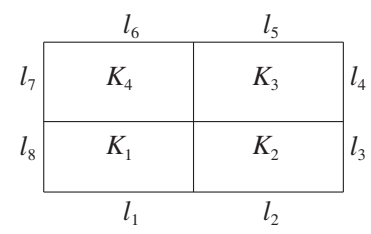

图 1 单元

Figure 1 Element

定理3 在与定理 2 同样的假设条件下, 可得

$$
\left\|I_{2 h} U^{n}-u^{n}\right\|_{1}=O\left(h^{2}+\tau^{\min \left\{2-\alpha_{1}, 3-\alpha\right\}}\right) .
$$

证明 由定理 2 和插值后处理算子 $I_{2 h}$ 的性质, 可得

$$
\begin{aligned}
\left\|I_{2 h} U^{n}-u^{n}\right\|_{1} & \leqslant\left\|I_{2 h} U^{n}-I_{2 h} I_{h} u^{n}\right\|_{1}+\left\|I_{2 h} I_{h} u^{n}-u^{n}\right\|_{1} \\
& =\left\|I_{2 h}\left(U^{n}-I_{h} u^{n}\right)\right\|_{1}+\left\|I_{2 h} u^{n}-u^{n}\right\|_{1} \\
& \leqslant C\left\|U^{n}-I_{h} u^{n}\right\|_{1}+C h^{2}\left\|u^{n}\right\|_{3} \\
& =O\left(h^{2}+\tau^{\min \left\{2-\alpha_{1}, 3-\alpha\right\}}\right) .
\end{aligned}
$$

\section{5 数值结果}

本节借助于一些数值算例从数值模拟的角度进一步展示理论结果的正确性和高效性.

例1 考虑如下两项时间混合分数阶扩散波动方程:

$$
\begin{cases}D_{t}^{\alpha_{1}} u(\boldsymbol{x}, t)+D_{t}^{\alpha} u(\boldsymbol{x}, t)-\Delta u(\boldsymbol{x}, t)=f(\boldsymbol{x}, t), & (\boldsymbol{x}, t) \in \Omega \times(0, T], \\ u(\boldsymbol{x}, t)=0, & (\boldsymbol{x}, t) \in \partial \Omega \times(0, T], \\ u(\boldsymbol{x}, 0)=0, & \boldsymbol{x} \in \Omega,\end{cases}
$$

其中 $\Omega=(0, \pi) \times(0, \pi), T=1$ 和 $f(\boldsymbol{x}, t)=\left[\frac{2 t^{2-\alpha_{1}}}{\Gamma\left(3-\alpha_{1}\right)}+\frac{6 t^{3-\alpha_{1}}}{\Gamma\left(4-\alpha_{1}\right)}+\frac{2 t^{2-\alpha}}{\Gamma(3-\alpha)}+\frac{6 t^{3-\alpha}}{\Gamma(4-\alpha)}+2\left(t^{2}+t^{3}\right)\right] \sin x \sin y$.

例2 在 (1) 式中, 选择不同的源项 $f(\boldsymbol{x}, t)=\left[\frac{\Gamma\left(2+\alpha_{1}+\alpha\right)}{\Gamma\left(2+\alpha_{1}\right)} t^{1+\alpha_{1}}+\frac{\Gamma\left(2+\alpha_{1}+\alpha\right)}{\Gamma(2+\alpha)} t^{1+\alpha}+2 t^{1+\alpha_{1}+\alpha}\right] \sin x \sin y$, 进行相关的数值模拟.

在表 1 中, 通过选取 $h=\tau^{\min \left\{2-\alpha_{1}, 3-\alpha\right\}}$ 和 $h^{2}=\tau^{\min \left\{2-\alpha_{1}, 3-\alpha\right\}}$, 我们给出了在 $t_{n}=1, \alpha_{1}=$ 0.3, 0.5, $\alpha=1.9,1.3$ 时, $\left\|u^{n}-U^{n}\right\|_{0},\left\|I_{h} u^{n}-U^{n}\right\|_{1},\left\|u^{n}-I_{2 h} U^{n}\right\|_{1}$ 和 $\left\|u^{n}-U^{n}\right\|_{1}$ 的时间方向上的最 优误差估计. 从表 1 中很容易看出 $\left\|u^{n}-U^{n}\right\|_{0},\left\|u^{n}-U^{n}\right\|_{1},\left\|I_{h} u^{n}-U^{n}\right\|_{1}$ 和 $\left\|u^{n}-I_{2 h} U^{n}\right\|_{1}$ 的时间收 玫阶接近于 $\min \left\{2-\alpha_{1}, 3-\alpha\right\}$, 这与理论分析相吻合.

在以上例子中, 我们用 $m, n$ 表示 $x$ 方向和 $y$ 方向的单元剖分数目, 然后分别取 $h=\pi \sqrt{\frac{1}{m^{2}}+\frac{1}{n^{2}}}$, $\tau=10^{-3}$. 例 1 中, 在 $\alpha_{1}=0.5, \alpha=1.5$ 时, $\left\|u^{n}-U^{n}\right\|_{0},\left\|I_{h} u^{n}-U^{n}\right\|_{1},\left\|u^{n}-I_{2 h} U^{n}\right\|_{1}$ 和 $\left\|u^{n}-U^{n}\right\|_{1}$ 在不 同时间的误差和收玫阶见表 2. 例 2 中, 在 $\alpha_{1}=0.1, \alpha=1.3$ 时, $\left\|u^{n}-U^{n}\right\|_{0},\left\|I_{h} u^{n}-U^{n}\right\|_{1},\left\|u^{n}-I_{2 h} U^{n}\right\|_{1}$ 和 $\left\|u^{n}-U^{n}\right\|_{1}$ 在不同时间的误差和收玫阶见表 3. 从表 2 和 3 中, 很容易看出 $\left\|u^{n}-U^{n}\right\|_{0},\left\|I_{h} u^{n}-U^{n}\right\|_{1}$ 和 $\left\|u^{n}-I_{2 h} U^{n}\right\|_{1}$ 的空间收敛阶接近 $2,\left\|u^{n}-U^{n}\right\|_{1}$ 的空间收玫阶接近于 1. 综上可知, 收敛、超逼近 和超收玫的数值结果都与理论分析相一致. 
表 1 在 $t_{n}=1, \alpha_{1}=0.3,0.5, \alpha=1.9,1.3$ 时时间方向的数值结果

Table 1 Temporal numerical results with $\alpha_{1}=0.3,0.5, \alpha=1.9,1.3$ at $t_{n}=1$

\begin{tabular}{cccccccccc}
\hline$\alpha_{1}, \alpha$ & $\tau$ & $\left\|u^{n}-U^{n}\right\|_{0}$ & Rate & $\left\|u^{n}-U^{n}\right\|_{1}$ & Rate & $\left\|I_{h} u^{n}-U^{n}\right\|_{1}$ & Rate & $\left\|u^{n}-I_{2 h} U^{n}\right\|_{1}$ & Rate \\
\hline \multirow{2}{*}{$\alpha_{1}=0.3$} & $t_{n} / 3$ & $4.373 \mathrm{E}-1$ & - & $8.276 \mathrm{E}-1$ & - & $8.978 \mathrm{E}-1$ & - & $9.202 \mathrm{E}-1$ & - \\
$\alpha=1.9$ & $t_{n} / 6$ & $2.140 \mathrm{E}-1$ & 1.031 & $3.985 \mathrm{E}-1$ & 1.054 & $4.327 \mathrm{E}-1$ & 1.053 & $4.387 \mathrm{E}-1$ & 1.069 \\
& $t_{n} / 12$ & $1.017 \mathrm{E}-1$ & 1.073 & $1.879 \mathrm{E}-1$ & 1.084 & $2.037 \mathrm{E}-1$ & 1.087 & $2.054 \mathrm{E}-1$ & 1.094 \\
& $t_{n} / 24$ & $4.768 \mathrm{E}-2$ & 1.093 & $8.793 \mathrm{E}-2$ & 1.096 & $9.574 \mathrm{E}-2$ & 1.089 & $9.639 \mathrm{E}-2$ & 1.092 \\
\hline \multirow{2}{*}{$\alpha_{1}=0.5$} & $t_{n} / 3$ & $1.619 \mathrm{E}-1$ & - & $3.377 \mathrm{E}-1$ & - & $3.536 \mathrm{E}-1$ & - & $3.378 \mathrm{E}-1$ & - \\
$\alpha=1.3$ & $t_{n} / 6$ & $5.854 \mathrm{E}-2$ & 1.467 & $1.214 \mathrm{E}-1$ & 1.476 & $1.287 \mathrm{E}-1$ & 1.458 & $1.307 \mathrm{E}-1$ & 1.370 \\
& $t_{n} / 12$ & $2.069 \mathrm{E}-2$ & 1.501 & $4.297 \mathrm{E}-2$ & 1.499 & $4.632 \mathrm{E}-2$ & 1.474 & $4.592 \mathrm{E}-2$ & 1.509 \\
& $t_{n} / 24$ & $7.242 \mathrm{E}-3$ & 1.515 & $1.506 \mathrm{E}-2$ & 1.496 & $1.622 \mathrm{E}-2$ & 1.514 & $1.622 \mathrm{E}-2$ & 1.505 \\
\hline
\end{tabular}

表 2 在 $t=0.3,0.5,0.9,1, \alpha=1.5, \alpha_{1}=0.5, \tau=10^{-3}$ 时空间数值结果

Table 2 Spatial numerical results with $\alpha=1.5, \alpha_{1}=0.5, \tau=10^{-3}$ at $t=0.3,0.5,0.9,1$

\begin{tabular}{cccccccccc}
\hline$t$ & $m \times n$ & $\left\|u^{n}-U^{n}\right\|_{0}$ & Rate & $\left\|u^{n}-U^{n}\right\|_{1}$ & Rate & $\left\|I_{h} u^{n}-U^{n}\right\|_{1}$ & Rate & $\left\|u^{n}-I_{2 h} U^{n}\right\|_{1}$ & Rate \\
\hline \multirow{4}{*}{$t=0.3$} & $4 \times 4$ & $6.434 \mathrm{E}-4$ & - & $6.034 \mathrm{E}-3$ & - & $3.063 \mathrm{E}-3$ & - & $3.954 \mathrm{E}-3$ & - \\
& $8 \times 8$ & $1.525 \mathrm{E}-4$ & 2.077 & $2.964 \mathrm{E}-3$ & 1.026 & $7.922 \mathrm{E}-4$ & 1.951 & $9.969 \mathrm{E}-4$ & 1.987 \\
& $16 \times 16$ & $3.755 \mathrm{E}-5$ & 2.021 & $1.475 \mathrm{E}-3$ & 1.006 & $2.009 \mathrm{E}-4$ & 1.979 & $2.507 \mathrm{E}-4$ & 1.992 \\
& $32 \times 32$ & $9.352 \mathrm{E}-6$ & 2.006 & $7.369 \mathrm{E}-4$ & 1.002 & $5.168 \mathrm{E}-5$ & 1.959 & $6.378 \mathrm{E}-5$ & 1.975 \\
\hline \multirow{4}{*}{$t=0.5$} & $4 \times 4$ & $2.076 \mathrm{E}-3$ & - & $1.930 \mathrm{E}-2$ & - & $9.576 \mathrm{E}-3$ & - & $1.247 \mathrm{E}-2$ & - \\
& $8 \times 8$ & $4.923 \mathrm{E}-4$ & 2.076 & $9.495 \mathrm{E}-3$ & 1.024 & $2.478 \mathrm{E}-3$ & 1.950 & $3.146 \mathrm{E}-3$ & 1.986 \\
& $16 \times 16$ & $1.212 \mathrm{E}-4$ & 2.022 & $4.728 \mathrm{E}-3$ & 1.006 & $6.273 \mathrm{E}-4$ & 1.982 & $7.900 \mathrm{E}-4$ & 1.993 \\
& $32 \times 32$ & $3.004 \mathrm{E}-5$ & 2.012 & $2.362 \mathrm{E}-3$ & 1.001 & $1.598 \mathrm{E}-4$ & 1.973 & $1.997 \mathrm{E}-4$ & 1.984 \\
\hline \multirow{3}{*}{$t=0.9$} & $4 \times 4$ & $8.746 \mathrm{E}-3$ & - & $7.893 \mathrm{E}-2$ & - & $3.731 \mathrm{E}-2$ & - & $4.951 \mathrm{E}-2$ & - \\
& $8 \times 8$ & $2.079 \mathrm{E}-3$ & 2.073 & $3.893 \mathrm{E}-2$ & 1.020 & $9.676 \mathrm{E}-3$ & 1.947 & $1.252 \mathrm{E}-2$ & 1.984 \\
& $16 \times 16$ & $5.122 \mathrm{E}-4$ & 2.021 & $1.939 \mathrm{E}-2$ & 1.005 & $2.446 \mathrm{E}-3$ & 1.984 & $3.140 \mathrm{E}-3$ & 1.995 \\
& $32 \times 32$ & $1.268 \mathrm{E}-4$ & 2.014 & $9.692 \mathrm{E}-3$ & 1.001 & $6.181 \mathrm{E}-4$ & 1.984 & $7.897 \mathrm{E}-4$ & 1.992 \\
\hline \multirow{2}{*}{$t=1$} & $4 \times 4$ & $1.146 \mathrm{E}-2$ & - & $1.024 \mathrm{E}-1$ & - & $4.787 \mathrm{E}-2$ & - & $6.384 \mathrm{E}-2$ & - \\
& $8 \times 8$ & $2.726 \mathrm{E}-3$ & 2.072 & $5.058 \mathrm{E}-2$ & 1.019 & $1.242 \mathrm{E}-2$ & 1.946 & $1.614 \mathrm{E}-2$ & 1.983 \\
& $16 \times 16$ & $6.719 \mathrm{E}-4$ & 2.020 & $2.521 \mathrm{E}-2$ & 1.005 & $3.139 \mathrm{E}-3$ & 1.984 & $4.051 \mathrm{E}-3$ & 1.995 \\
& $32 \times 32$ & $1.665 \mathrm{E}-4$ & 2.013 & $1.259 \mathrm{E}-2$ & 1.001 & $7.926 \mathrm{E}-4$ & 1.946 & $1.018 \mathrm{E}-3$ & 1.993 \\
\hline
\end{tabular}

\section{6 总结与展望}

本文基于双线性有限元方法和 L1-CN 格式, 分析了两项时间混合分数阶扩散波动方程全离散逼 近格式的稳定性和收玫性, 得到了超逼近和超收玫结果. 首先, 证明了全离散逼近格式是无条件稳定 的. 其次, 用双线性元的高精度结果和一些引理得到了收玫性和超逼近结果. 然后, 利用插值后处理技 术, 得到了 $H^{1}$ 模意义下的整体超收玫结果 $O\left(h^{2}+\tau^{\min \left\{2-\alpha_{1}, 3-\alpha\right\}}\right)$, 并且对其他单元应用于该方程的 数值分析做了进一步讨论. 最后, 借助于数值算例进一步展示了理论分析的正确性. 本文的创新之处 在于不仅用有限元方法对二维两项时间混合分数阶扩散波动方程进行了稳定性和收玫性分析, 而且还 得到了超收玫结果. 近期, 我们考虑用这种高效的数值方法来研究更多类型的空间分数阶微分方程和 
表 3 在 $t=0.2,0.4,0.6,0.8, \alpha=1.3, \alpha_{1}=0.1, \tau=10^{-3}$ 时空间方向的数值结果

Table 3 Spatial numerical results with $\alpha=1.3, \alpha_{1}=0.1, \tau=10^{-3}$ at $t=0.2,0.4,0.6,0.8$

\begin{tabular}{cccccccccc}
\hline$t$ & $m \times n$ & $\left\|u^{n}-U^{n}\right\|_{0}$ & Rate & $\left\|u^{n}-U^{n}\right\|_{1}$ & Rate & $\left\|I_{h} u^{n}-U^{n}\right\|_{1}$ & Rate & $\left\|u^{n}-I_{2 h} U^{n}\right\|_{1}$ & Rate \\
\hline \multirow{4}{*}{$t=0.2$} & $4 \times 4$ & $1.155 \mathrm{E}-3$ & - & $1.084 \mathrm{E}-2$ & - & $5.508 \mathrm{E}-3$ & - & $7.106 \mathrm{E}-3$ & - \\
& $8 \times 8$ & $2.738 \mathrm{E}-4$ & 2.077 & $5.323 \mathrm{E}-3$ & 1.026 & $1.424 \mathrm{E}-3$ & 1.951 & $1.792 \mathrm{E}-3$ & 1.988 \\
& $16 \times 16$ & $6.744 \mathrm{E}-5$ & 2.021 & $2.649 \mathrm{E}-3$ & 1.006 & $3.611 \mathrm{E}-4$ & 1.979 & $4.504 \mathrm{E}-4$ & 1.992 \\
& $32 \times 32$ & $1.679 \mathrm{E}-5$ & 2.006 & $1.323 \mathrm{E}-3$ & 1.002 & $9.267 \mathrm{E}-5$ & 1.962 & $1.144 \mathrm{E}-4$ & 1.977 \\
\hline \multirow{4}{*}{$t=0.4$} & $4 \times 4$ & $6.156 \mathrm{E}-3$ & - & $5.706 \mathrm{E}-2$ & - & $2.813 \mathrm{E}-2$ & - & $3.671 \mathrm{E}-2$ & - \\
& $8 \times 8$ & $1.460 \mathrm{E}-3$ & 2.076 & $2.808 \mathrm{E}-2$ & 1.023 & $7.277 \mathrm{E}-3$ & 1.950 & $9.261 \mathrm{E}-3$ & 1.987 \\
& $16 \times 16$ & $3.599 \mathrm{E}-4$ & 2.020 & $1.398 \mathrm{E}-2$ & 1.006 & $1.837 \mathrm{E}-3$ & 1.986 & $2.322 \mathrm{E}-3$ & 1.996 \\
& $32 \times 32$ & $8.944 \mathrm{E}-5$ & 2.009 & $6.984 \mathrm{E}-3$ & 1.001 & $4.631 \mathrm{E}-4$ & 1.988 & $5.831 \mathrm{E}-4$ & 1.994 \\
\hline \multirow{3}{*}{$t=0.6$} & $4 \times 4$ & $1.656 \mathrm{E}-2$ & - & $1.506 \mathrm{E}-1$ & - & $7.195 \mathrm{E}-2$ & - & $9.508 \mathrm{E}-2$ & - \\
& $8 \times 8$ & $3.935 \mathrm{E}-3$ & 2.073 & $7.425 \mathrm{E}-2$ & 1.021 & $1.864 \mathrm{E}-2$ & 1.948 & $2.402 \mathrm{E}-2$ & 1.985 \\
& $16 \times 16$ & $9.707 \mathrm{E}-4$ & 2.019 & $3.699 \mathrm{E}-2$ & 1.005 & $4.705 \mathrm{E}-3$ & 1.987 & $6.021 \mathrm{E}-3$ & 1.996 \\
& $32 \times 32$ & $2.415 \mathrm{E}-4$ & 2.007 & $1.848 \mathrm{E}-2$ & 1.001 & $1.182 \mathrm{E}-3$ & 1.993 & $1.508 \mathrm{E}-3$ & 1.997 \\
\hline \multirow{2}{*}{$t=0.8$} & $4 \times 4$ & $3.374 \mathrm{E}-2$ & - & $2.998 \mathrm{E}-1$ & - & $1.389 \mathrm{E}-1$ & - & $1.859 \mathrm{E}-1$ & - \\
& $8 \times 8$ & $8.033 \mathrm{E}-3$ & 2.071 & $1.480 \mathrm{E}-1$ & 1.018 & $3.606 \mathrm{E}-2$ & 1.945 & $4.701 \mathrm{E}-2$ & 1.983 \\
& $16 \times 16$ & $1.983 \mathrm{E}-3$ & 2.018 & $7.378 \mathrm{E}-2$ & 1.004 & $9.099 \mathrm{E}-3$ & 1.987 & $1.178 \mathrm{E}-2$ & 1.996 \\
& $32 \times 32$ & $4.936 \mathrm{E}-4$ & 2.006 & $3.686 \mathrm{E}-2$ & 1.001 & $2.283 \mathrm{E}-3$ & 1.995 & $2.950 \mathrm{E}-3$ & 1.998 \\
\hline
\end{tabular}

时空分数阶微分方程. 另外, 对于其他有限单元 (包括协调元和非协调元) 应用于方程 (1) 的数值分析 情况, 也进行了简要的总结.

(i) 若将 $V_{0}^{h}$ 换成线性三角形有限元空间, 由于该元有如下性质: 设 $u \in H^{3}(\Omega)$, 则

$$
\left(\nabla\left(u-I_{h} u\right), \nabla v^{h}\right)=O\left(h^{2}\right)|u|_{3}\left|v^{h}\right|_{1}, \quad \forall v^{h} \in V_{0}^{h} .
$$

该单元不满足引理 1 的结果, 因此利用它无法得到定理 2 和 3 的结果.

(ii) 若将 $V_{0}^{h}$ 换成非常规 Hermite 矩形有限元空间, 在文献 [46] 中已经证明了如下结果: 设 $u \in$ $H^{4}(\Omega)$, 则

$$
\left(\nabla\left(u-I_{h} u\right), \nabla v^{h}\right)=O\left(h^{3}\right)|u|_{4}\left\|v^{h}\right\|_{1}, \quad \forall v^{h} \in V_{0}^{h},
$$

利用逆不等式, 可以得到

$$
\left(\nabla\left(u-I_{h} u\right), \nabla v^{h}\right)=O\left(h^{2}\right)|u|_{4}\left\|v^{h}\right\|_{0}, \quad \forall v^{h} \in V_{0}^{h} .
$$

因此, 利用该单元无法得到超逼近和超收玫结果.

(iii) 若将 $V_{0}^{h}$ 换成矩形网格下文献 [47 52] 中的 $E Q_{1}^{\text {rot }}$ 非协调元或文献 [53] 中正方形网格下的 $Q_{1}^{\text {rot }}$ 非协调元或文献 [54] 中带约束的旋转 $Q_{1}$ 元 (等价于矩形网格上文献 [55] 中的 $P_{1}$ 非协调元) 或 文献 [56] 中的类 Carey 元空间. 由于上述这些非协调元的相容误差只能估计为

$$
\left|\sum_{k} \int_{\partial k} \frac{\partial u}{\partial \boldsymbol{n}} v^{h} \mathrm{~d} s\right|=O\left(h^{2}\right)\|u\|_{3}\left\|v^{h}\right\|_{h}=O(h)\|u\|_{3}\left\|v^{h}\right\|_{0}, \quad \forall v^{h} \in V_{0}^{h},
$$

其中 $\|\cdot\|_{h}=\left(\sum_{k}|\cdot|_{1, k}^{2}\right)^{\frac{1}{2}}$ 是 $V_{0}^{h}$ 上的模. 因此, 利用它们, 到目前还无法得到定理 2 和 3 的结果. 
对四边形网格来说, 由于文献 $[57,58]$ 中关于 $E Q_{1}^{\text {rot }}$ 元的插值误差和相容误差只有 $O(h)$ 阶, 如何 将它应用于本文并得到超逼近和超收敛性也有待进一步研究.

(iv) 对文献 [59,60] 中的类 Wilson 元来说, 在任意四边形网格下, 当问题的精确解 $u \in H^{3}(\Omega)$ 时, 利用该元的相容误差在能量模意义下可以达到 $O\left(h^{2}\right)$ 阶, 即比插值误差高一阶的特殊性质, 类似 (iii) 的讨论, 利用它也不能得到定理 2 和 3 的结果. 对于矩形网格下的类 Wilson 元 ${ }^{[61]}$, 广义网格下改进 的类 Wilson 元 ${ }^{[62]}$ 和三角形剖分下的 $p_{1}^{\bmod }$ 元 ${ }^{[63]}$, 因为相容误差高达 $O\left(h^{3}\right)$ 阶, 因此由它们可以得到 本文的定理 2 和 3 , 但此时要求 $u \in H^{4}(\Omega)$, 显然要求的光滑度较高.

(v) 对于著名的 Wilson 元 ${ }^{[64]}$ 和 Carey 元 ${ }^{[65]}$, 因其相容误差仅有 $O(h)$ 阶, 到目前为止也仍无法 推出本文定理 2 和 3 的结果.

\section{参考文献}

1 Oldham K B, Spanier J. The Fractional Calculus. New York: Academic Press, 1974

2 Kosztołowicz T. Subdiffusion in a system with a thick membrane. J Membrane Sci, 2008, 320: 492-499

3 Chen J, Liu F W, Turner I, et al. The fundamental and numerical solutions of the Riesz space-fractional reactiondispersion equation. ANZ J, 2008, 50: 45-57

4 Zhuang P H, Liu F W, Anh V, et al. New solution and analytical techniques of the implicit numerical method for the anomalous subdiffusion equation. SIAM J Numer Anal, 2008, 46: 1079-1095

5 Podlubny I. Fractional Differential Equations. Manhattan: Academic Press, 1999

6 Murio D A. Implicit finite difference approximation for time fractional diffusion equations. Comput Math Appl, 2008, 56: $1138-1145$

7 Zhang Q, Zhang J W, Jiang S D, et al. Numerical solution to a linearized time fractional KdV equation on unbounded domains. Math Comput, 2018, 87: 693-719

8 Zeng F H, Li C P, Liu F W, et al. Numerical hms for time-fractional subdiffusion equation with second-order accuracy. SIAM J Sci Comput, 2015, 37: A55-A78

9 Liu Y, Yu Z D, Li H, et al. Time two-mesh algorithm combined with finite element method for time fractional water wave model. Int J Heat Mass Transfer, 2018, 120: 1132-1145

10 Jiang Y J, Ma J T. High-order finite element methods for time-fractional partial differential equations. J Comput Appl Math, 2011, 235: 3285-3290

11 Wang F L, Liu F W, Zhao Y M, et al. A novel approach of high accuracy analysis of anisotropic bilinear finite element for time-fractional diffusion equations with variable coefficient. Comput Math Appl, 2018, 75: 3786-3800

12 Yang J Y, Zhao Y M, Liu N, et al. An implicit MLS meshless method for 2-D time dependent fractional diffusion-wave equation. Appl Math Model, 2015, 39: 1229-1240

13 Mardani A, Hooshmandasl M R, Heydari M H, et al. A meshless method for solving the time fractional advectiondiffusion equation with variable coefficients. Comput Math Appl, 2018, 75: 122-133

14 Shivanian E, Jafarabadi A. Analysis of the spectral meshless radial point interpolation for solving fractional reactionsubdiffusion equation. J Comput Appl Math, 2018, 336: 98-113

15 Mustapha K, Abdallah B, Furati K M. A discontinuous Petrov-Galerkin method for time-fractional diffusion equations. SIAM J Numer Anal, 2014, 52: 2512-2529

16 Zhao Y M, Chen P, Bu W P, et al. Two mixed finite element methods for time-fractional diffusion equations. J Sci Comput, 2017, 70: 407-428

17 Li X J, Xu C J. A space-time spectral method for the time fractional diffusion equation. SIAM J Numer Anal, 2009, 47: 2108-2131

18 Liu Q X, Gu Y T, Zhuang P H, et al. An implicit RBF meshless approach for time fractional diffusion equations. Comput Mech, 2011, 48: 1-12

19 Lin Y M, Xu C J. Finite difference/spectral approximations for the time-fractional diffusion equation. J Comput Phys, 2007, 225: 1533-1552

20 Shi Z G, Zhao Y M, Liu F W, et al. Superconverse analysis of an $\mathrm{H}^{1}$-Galerkin mixed finite element method for 
two-dimensional multi-term time fractional diffusion equations. Comput Math Appl, 2017, 74: 1903-1914

21 Jin B T, Lazarov R, Liu Y K, et al. The Galerkin finite element method for a multi-term time-fractional diffusion equation. J Comput Phys, 2015, 281: 825-843

22 Zheng M, Liu F W, Anh V, et al. A high-order spectral method for the multi-term time-fractional diffusion equations. Appl Math Model, 2016, 40: 4970-4985

23 Zhao Y M, Zhang Y D, Liu F W, et al. Convergence and superconvergence of a fully-discrete scheme for multi-term time fractional diffusion equations. Comput Math Appl, 2017, 73: 1087-1099

24 Zhao Y M, Zhang Y D, Liu F W, et al. Analytical solution and nonconforming finite element approximation for the 2D multi-term fractional subdiffusion equation. Appl Math Model, 2016, 40: 8810-8825

25 Carcione J M, Cavallini F, Mainardi F, et al. Time-domain modeling of constant-Q seismic waves using fractional derivatives. Pure Appl Geophys, 2002, 159: 1719-1736

26 Sun Z Z, Wu X N. A fully discrete difference scheme for a diffusion-wave system. Appl Numer Math, 2006, 56: 193-209

27 Fairweather G, Yang X H, Xu D, et al. An ADI Crank-Nicolson orthogonal spline collocation method for the twodimensional fractional diffusion-wave equation. J Sci Comput, 2015, 65: 1217-1239

28 Huang J F, Tang Y F, Vázquez L, et al. Two finite difference schemes for time fractional diffusion-wave equation. Numer Algor, 2013, 64: 707-720

29 Du R, Cao W R, Sun Z Z. A compact difference scheme for the fractional diffusion-wave equation. Appl Math Model, 2010, 34: 2998-3007

30 Jin B T, Lazarov R, Zhou Z. Two fully discrete schemes for fractional diffusion and diffusion-wave equations with nonsmooth data. SIAM J Sci Comput, 2016, 38: A146-A170

31 Li L M, Xu D, Luo M. Alternating direction implicit Galerkin finite element method for the two-dimensional fractional diffusion-wave equation. J Comput Phys, 2013, 255: 471-485

32 Bhrawy A H, Doha E H, Baleanu D, et al. A spectral tau algorithm based on Jacobi operational matrix for numerical solution of time fractional diffusion-wave equations. J Comput Phys, 2015, 293: 142-156

33 Zhang Y D, Zhao Y M, Wang F L, et al. High-accuracy finite element method for 2D time fractional diffusion-wave equation on anisotropic meshes. Int J Comput Math, 2018, 95: 218-230

34 Liu F W, Meerschaert M M, McGough R, et al. Numerical methods for solving the multi-term time fractional wave equations. Fract Calc Appl Anal, 2013, 16: 9-25

35 Kilbas A A, Srivastava H M, Trujillo J J. Theory and Applications of Fractional Differential Equations. Amsterdam: Elsevier, 2006

36 Li M X, Guan X F, Mao S P. Convergence and superconvergence analysis of lagrange rectangular elements with any order on arbitrary rectangular meshes. J Comput Math, 2014, 32: 169-182

37 Huang Y Q, Li J C, Wu C, et al. Superconvergence analysis for linear tetrahedral edge elements. J Sci Comput, 2015, 62: 122-145

38 Shi D Y, Wang P L, Zhao Y M. Superconvergence analysis of anisotropic linear triangular finite element for nonlinear Schrödinger equation. Appl Math Lett, 2014, 38: 129-134

39 Lin Q, Lin J F. Finite Element Methods: Accuracy and Improvement. Beijing: Science Press, 2006

40 Lin Q, Yan N N. The Construction and Analysis of High Efficiency Finite Element Methods. Baoding: HeBei University Press, 1996

41 Sun Z Z. The Method of Order Reduction and Its Application to the Numerical Solutions of Partial Differential Equations. Beijing: Science Press, 2009

42 López-Marcos J C. A difference scheme for a nonlinear partial integrodifferential equation. SIAM J Numer Anal, 1990, 27: 20-31

43 Zhang Y N, Sun Z Z, Zhao X. Compact alternating direction implicit scheme for the two-dimensional fractional diffusion-wave equation. SIAM J Numer Anal, 2012, 50: 1535-1555

44 Ren J C, Sun Z Z. Numerical algorithm with high spatial accuracy for the fractional diffusion-wave equation with neumann boundary conditions. J Sci Comput, 2013, 56: 381-408

45 Feng L B, Liu F W, Turner I. Novel numerical analysis of multi-term time fractional viscoelastic non-Newtonian fluid models for simulating unsteady MHD Couette flow of a generalized Oldroyd-B fluid. ArXiv: 1710.09976, 2017

46 Shi D Y, Liang H. A new unconventional Hermite type anisotropic rectangular element superconvergence analysis and 
extrapolation. J Comput Math, 2005, 27: 369-382

47 Shi D Y, Wang F L, Shi Y H. The general format of the high accuracy analysis of the anisotropic $E Q_{1}^{r o t}$ nonconforming element. J Comput Math, 2013, 35: 239-252

48 Lin Q, Tobiska L, Zhou A H. Superconvergence and extrapolation of non-conforming low order finite elements applied to the Poisson equation. IMA J Numer Anal, 2005, 25: 160-181

49 Shi D Y, Mao S P, Chen S C. An anisotropic nonconforming finite element with some superconvergence results. J Comput Math, 2005, 23: 261-274

50 Shi D Y, Wang H H, Du Y P. An anisotropic nonconforming finite element method for approximating a class of nonlinear Sobolev equations. J Comput Math, 2009, 27: 299-314

51 Shi D Y, Zhou J Q, Shi D W. A new low order least squares nonconforming characteristics mixed finite element method for Burgers' equation. Appl Math Comput, 2013, 219: 11302-11310

52 Shi D Y, Yu Z Y. Low-order nonconforming mixed finite element methods for stationary inconpressible magnetohydrodynamics equations. J Appl Math, 2013, 10: 904-919

53 Rannacher R, Turek S. Simple nonconforming quadrilateral Stokes element. Numer Methods Partial Differ Eq, 1992, 8: $97-111$

$54 \mathrm{Hu}$ J, Meng H Y, Shi Z C. Application of constrained nonconforming rotational $Q_{1}$-element in stokes and plane elastic problems. J Comput Math, 2005, 27: 311-324

55 Park C J, Sheen D W. $P_{1}$-nonconforming quadrilateral finite element method for second order elliptic problems. SIAM J Numer Anal, 2003, 41: 624-640

56 Shi D Y, Hao X B. Accuracy analysis for quasi-Carey element. J Syst Sci Complex, 2008, 21: 456-462

57 Shi D Y, Xu C, Chen J H. Anisotropic nonconforming $E Q_{1}^{\text {rot }}$ quadrilateral finite element approximation to second order elliptic problems. J Sci Comput, 2013, 56: 637-653

58 Shi D Y, Xu C. EQ $Q_{1}^{\text {rot }}$ nonconforming finite element approximation to Signorini problem. Sci China Math, 2013, 56: $1301-1311$

59 Chen S C, Shi D Y. Accuracy analysis for quasi-Wilson element. Acta Math Sci, 2000, 20: 44-48

60 Chen S C, Shi D Y, Zhao Y C. Anisotropic interpolation and quasi-Wilson element for narrow quadrilateral meshes. IMA J Numer Anal, 2004, 24: 77-95

61 Shi D Y, Wang F L, Zhao Y M. Superconvergence analysis and extrapolation of quasi-Wilson nonconforming finite element method for nonlinear Sobolev equations. Acta Math Appl Sin Engl Ser, 2013, 29: 403-414

62 Shi D Y, Pei L F. Nonconforming quadrilateral finite element method for a class of nonlinear sine-Gorden equations. Appl Math Comput, 2013, 219: 9447-9460

63 Knobloch P, Tobiska L. The $P_{1}^{\text {mod }}$ element: a new nonconforming finite element for convection diffusion problems. SIAM J Numer Anal, 2003, 41: 436-456

64 Shi Z C, Jiang B, Xue W M. A new superconvergence property of Wilson nonconforming finite element. Numerische Mathematik, 1997, 78: 259-268

65 Shi D Y, Hao X B. High accuracy analysis of Sobolev-type equation anisotropic Carey element. J Eng Math, 2009, 29: $1021-1026$

\section{High-accuracy analysis of finite-element method for two-term mixed time-fractional diffusion-wave equations}

Yabing WEI ${ }^{1,2}$, Yanmin $\mathrm{ZHAO}^{1}$, Yifa TANG ${ }^{3,4^{*}}$, Fenling WANG ${ }^{1}$, Zhengguang $\mathrm{SHI}^{5}$ \& Kuangyin $\mathrm{LI}^{6}$

1. School of Mathematics and Statistics, Xuchang University, Xuchang 461000, China;

2. School of Mathematical and Statistics, Zhengzhou University, Zhengzhou 450001, China;

3. LSEC, ICMSEC, Academy of Mathematics and Systems Science, Chinese Academy of Sciences, Beijing 100190, China;

4. School of Mathematical Sciences, University of Chinese Academy of Sciences, Beijing 100049, China;

5. School of Economic Matnematics, Southwestern University of finance and Economic, Chengdu 611130, China;

6. McDougall School of Petroleum Engineering, The University of Tulsa, Tulsa, OK 74104, USA

* Corresponding author. E-mail: tyf@lsec.cc.ac.cn 


\begin{abstract}
Based on the finite-element method (FEM) in the spatial direction and L1-CN approximation in the temporal direction, respectively, numerical analyses are proposed for 2D two-term mixed time-fractional diffusionwave equations. First, a fully discrete approximate scheme is established for the equation, and it is proved to be unconditionally stable. Then, rigorous proofs are provided for the convergence result in the $L^{2}$ - norm and superclose properties in $H^{1}$ - norm with order $O\left(h^{2}+\tau^{\min \left\{2-\alpha_{1}, 3-\alpha\right\}}\right)\left(0<\alpha_{1}<1,1<\alpha<2\right)$, where $h$ and $\tau$ are the spatial size and time step, respectively. Furthermore, the global superconvergence in the $H^{1}-$ norm is obtained using an interpolation postprocessing technique. Finally, with the help of numerical examples, the correctness and high efficiency of the theoretical analysis are further demonstrated.
\end{abstract}

Keywords two-term mixed time-fractional diffusion-wave equations, finite-element method, L1-CN approximation, stability, superclose, convergence and superconvergence

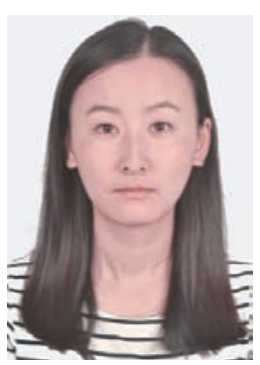

Yabing WEI was born in Xuchang, China, in 1990. She received a B.S. degree in mathematics and applied mathematics from Xuchang University in 2014. Currently, she is a graduate student in applied mathematics at Zhengzhou University. Her research interests include finite-element methods with applications.

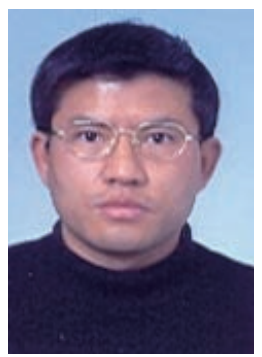

Yifa TANG received a Ph.D. degree in applied mathematics from the Institute of Computational Mathematics, Chinese Academy of Sciences, in 1997, where he was an assistant professor from July 1997 to October 1998 and an associate professor from November 1998 to January 1999. From February 1999, he has been with the Academy of Mathematics and Systems Science, Chinese Academy of Sciences, where he is currently a research professor of computational mathematics. His research interests include symplectic algorithms for Hamiltonian systems and numerical analysis of fractional differential equations with applications.

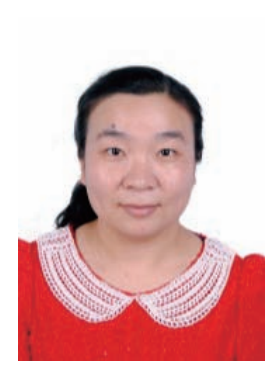

Yanmin ZHAO was born in Xuchang, China, in 1979. She received a B.S degree in mathematics education and an M.S. degree in computational mathematics from Henan University and Zhengzhou University, in 2002 and 2005, respectively, and a Ph.D. degree in applied mathematics from the Institute of Computational Mathematics, Chinese Academy of Sciences, Beijing, China in 2008. Currently, she is a professor with the School of Mathematics and Statistics, Xuchang University. Her research interests include symplectic algorithms for Hamiltonian systems and finiteelement methods with applications.

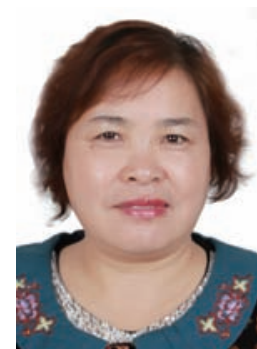

Fenling WANG was born in Xuchang, China, in 1968. She received a B.S. degree in mathematics education and M.S. degree in fundamental mathematics from Henan Institute of Education and Xinyang Normal University in 1999 and 2009, respectively. Currently, she is a professor at the School of Mathematics and Statistics, Xuchang University. Her research interests include finiteelement methods with applications. 